\title{
Slow translational instabilities of spike patterns in the one-dimensional Gray-Scott model
}

\author{
THEODORE KOLOKOLNIKOV ${ }^{\dagger}$ AND MiCHAEL J. WARD ${ }^{\ddagger}$ \\ Department of Mathematics, University of British Columbia, Vancouver, Canada V6T $1 Z 2$ \\ JUNCHENG WEI ${ }^{\S}$ \\ Department of Mathematics, Chinese University of Hong Kong, New Territories, Hong Kong
}

[Received 17 March 2005 and in revised form 7 February 2006]

\begin{abstract}
Slow translational instabilities of symmetric $k$-spike equilibria for the one-dimensional singularly perturbed two-component Gray-Scott (GS) model are analyzed. These symmetric spike patterns are characterized by a common value of the spike amplitude. The GS model is studied on a finite interval in the semi-strong spike-interaction regime, where the diffusion coefficient of only one of the two chemical species is asymptotically small. Two distinguished limits for the GS model are considered: the low feed-rate regime and the intermediate regime. In the low feed-rate regime it is shown analytically that $k-1$ small eigenvalues, governing the translational stability of a symmetric $k$ spike pattern, simultaneously cross through zero at precisely the same parameter value at which $k-1$ different asymmetric $k$-spike equilibria bifurcate off of the symmetric $k$-spike equilibrium branch. These asymmetric equilibria have the general form $S B B \ldots B S$ (neglecting the positioning of the $B$ and $S$ spikes in the overall spike sequence). For a one-spike equilibrium solution in the intermediate regime it is shown that a translational, or drift, instability can occur from a Hopf bifurcation in the spike-layer location when a reaction-time parameter $\tau$ is asymptotically large as $\varepsilon \rightarrow 0$. Locally, this instability leads to small-scale oscillations in the spike-layer location. For a certain parameter range within the intermediate regime such a drift instability for the GS model is shown to be the dominant instability mechanism. Numerical experiments are performed to validate the asymptotic theory.
\end{abstract}

\section{Introduction}

We study the translational stability of equilibrium spike solutions in the one-dimensional Gray-Scott (GS) model in particular parameter regimes. The GS system, introduced for continuously stirred systems in [14], models an irreversible reaction involving two reactants in a gel reactor, where the reactor is maintained in contact with a reservoir of one of the chemical species. The dimensionless GS model is (cf. [30], [21])

$$
\begin{aligned}
& v_{t}=v_{x x}-v+A u v^{2}, \quad-1<x<1, t>0 ; \quad v_{x}( \pm 1, t)=0 \text {, } \\
& \tau u_{t}=D u_{x x}+(1-u)-u v^{2}, \quad-1<x<1, t>0 ; \quad u_{x}( \pm 1, t)=0 \text {. }
\end{aligned}
$$

Here $A>0$ is the feed-rate parameter, $D>0, \tau>1$, and $0<\varepsilon \ll 1$. For $\varepsilon \ll 1$, there are equilibrium solutions for $v$, called spike patterns, that are localized near certain points in the domain.

\footnotetext{
${ }^{\dagger}$ E-mail: tkolokol@mathstat.dal.ca

‡E-mail: ward@math.ubc.ca

${ }^{\S}$ E-mail: wei@math.cuhk.edu.hk
} 
The parameter $D$ measures the strength of the inter-spike interactions. The resulting spike patterns can be classified into two main categories. The semi-strong spike interaction regime corresponds to the limit $\varepsilon^{2} \ll 1$ and $D=O(1)$. The weak-interaction regime, where both the $u$ and $v$ components are localized, corresponds to the parameter range $D=O\left(\varepsilon^{2}\right) \ll 1$. In this paper we will focus on the semi-strong regime.

The numerical study of [38] for the GS model in the weak-interaction regime in a twodimensional domain showed a plethora of spike-type patterns in certain parameter ranges, including time-dependent oscillating spikes, spike death due to over-crowding, spike-replication behavior, spatio-temporal chaos, labyrinthine patterns and zigzag instabilities, etc. The similarities between these behaviors and chemical patterns arising in certain physical experiments are striking (cf. [24], [25]). These numerical and experimental studies have stimulated much theoretical work to classify steady-state and time-dependent spike behavior in the simpler case of one spatial dimension, including: spike-replication and dynamics in the weak-interaction regime (cf. [39], [41], [36], [44]); spatio-temporal chaos in the weak-interaction regime (cf. [37]); the existence and stability of equilibrium solutions in the semi-strong interaction regime (cf. [11], [7], [8], [28], [29], [30], [21], [22]), and the dynamics of solutions in the semi-strong interaction regime (cf. [5], [6], [43]).

For (1.1) in the semi-strong interaction limit, there are three distinguished limits for $A$ where different behavior is observed: The low feed-rate regime $A=O\left(\varepsilon^{1 / 2}\right)$, the intermediate regime $O\left(\varepsilon^{1 / 2}\right) \ll A \ll 1$, and the high feed-rate regime $A=O(1)$. The high feed-rate regime is associated with pulse-splitting behavior (cf. [7], [30], [22]). In the low feed-rate regime, we introduce new variables $\mathcal{A}$ and $v$ defined by

$$
A=\varepsilon^{1 / 2} \mathcal{A}, \quad v=\varepsilon^{-1 / 2} \nu .
$$

In terms of $(1.2),(1.1)$ is transformed to

$$
\begin{aligned}
& v_{t}=\varepsilon^{2} v_{x x}-v+\mathcal{A} u v^{2}, \quad-1<x<1, t>0 ; \quad v_{x}( \pm 1, t)=0, \\
& \tau u_{t}=D u_{x x}+(1-u)-\varepsilon^{-1} u v^{2}, \quad-1<x<1, t>0 ; \quad u_{x}( \pm 1, t)=0 \text {. }
\end{aligned}
$$

In [21] symmetric $k$-spike equilibrium solutions to (1.3), where the spikes have a common amplitude, were constructed asymptotically in the low feed-rate regime. A construction of similar solutions using the geometric theory of singular perturbations is given in [11] for a special scaling of the GS model. In [21] the stability of $k$-spike patterns on an $O(1)$ time-scale was analyzed by first formally deriving a nonlocal eigenvalue problem (NLEP). From a rigorous analysis of this NLEP, two fast instability mechanisms were identified: synchronous oscillatory instabilities of the spike amplitudes, and competition, or overcrowding, instabilities leading to the spike annihilation events. In the intermediate regime $O(1) \ll \mathcal{A} \ll O\left(\varepsilon^{-1 / 2}\right)$, the existence and stability of $k$-spike patterns was first analyzed in [7] (see also $\S 4$ of [21] for a different approach). A stability analysis for a one-spike solution on the infinite line was given in [29] in both the low and intermediate feed-rate regimes. In $\S 2$ and Appendix A we briefly summarize those previous results for fast instabilities that are important for an understanding of the new results derived herein.

The goal here is to study slow translational instabilities of equilibrium spike solutions for the GS model in the low and intermediate feed-rate regimes. A related analysis for stripe and ring-type solutions to the GS model is given in [23]. For the low feed-rate regime $\mathcal{A}=O(1)$, in $\$ 3$ we analyze the stability of symmetric $k$-spike equilibrium solutions with respect to the small eigenvalues of 


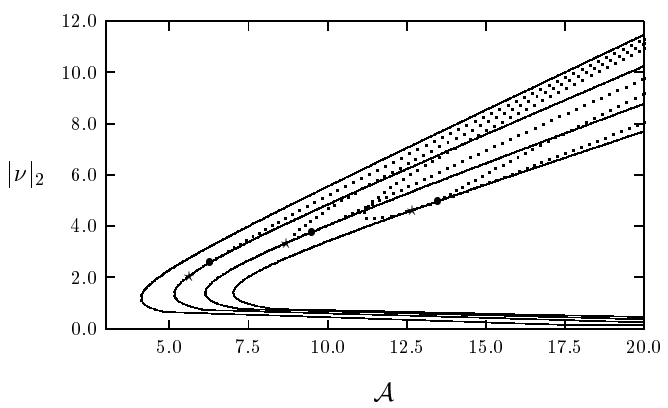

(a) Symmetric and asymmetric branches: $D=0.75$

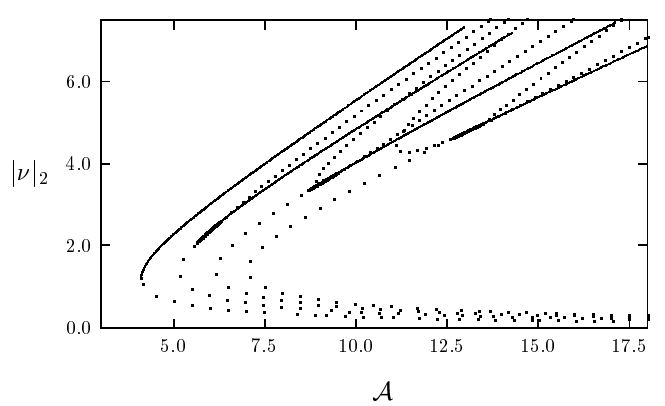

(b) Stability of symmetric branches: $D=0.75$

FIG. 1. Left figure: $|\nu|_{2}$ versus $\mathcal{A}$ for the symmetric (solid curves) and asymmetric (dotted curves) solution branches when $\mathcal{A}=O(1), D=0.75$ and $k=1,2,3,4$. The saddle-node values $\mathcal{A}_{k e}$ increase with $k$. Right figure: $|v|_{2}$ versus $\mathcal{A}$ stability plot. The smooth curve for each symmetric solution branch has three portions with different stability properties. The curves with widely spaced dots are unstable for $\tau \geqslant 0$. The heavy solid curves are stable only with respect to the large eigenvalues when $\tau<\tau_{h L}$. The solid curves are stable with respect to both the large and small eigenvalues when $\tau<\tau_{h L}$. The asymmetric branches, shown as the dotted curves, bifurcate at the point where the solid and heavy solid curves meet.

order $O\left(\varepsilon^{2}\right)$ that govern translational instabilities of the spike profile. Using a formal asymptotic analysis, in Principal Result 3.1 we obtain a certain auxiliary problem that the small eigenvalues satisfy. By solving this problem exactly, we give explicit formulae for the small eigenvalues in Proposition 3.2 and explicit stability thresholds with respect to $D$ and $\mathcal{A}$ in Propositions 3.3 and 3.4, respectively. In particular, in Proposition 3.4 we show for any $\tau=O(1), k>1$, and fixed $D>0$, that the $k$-spike pattern is stable with respect to translations only when $\mathcal{A}>\mathcal{A}_{k S}$, for some explicit threshold $\mathcal{A}_{k S}$. As $\mathcal{A}$ decreases below $\mathcal{A}_{k S}$, and for $k>1$, there are $k-1$ small eigenvalues that simultaneously cross into the unstable right half-plane $\operatorname{Re}(\lambda)>0$ along the real axis. Furthermore, for $\tau$ below some threshold and $k>1$, we show there is a range of values of $\mathcal{A}$ where a symmetric $k$-spike equilibrium pattern is unstable with respect to translations but is stable with respect to the large eigenvalues of the NLEP that govern fast $O(1)$ instabilities. For $D=0.75$, this range of $\mathcal{A}$ is illustrated in Fig. 1(b) In $\$ 3.1$ we perform numerical computations to illustrate spike dynamics for (1.3) in this range of $\mathcal{A}$.

In $\S 4$ we use a formal asymptotic analysis to study the existence of asymmetric $k$-spike patterns where the spikes have different heights. In Principal Result 4.2 we show that the resulting spike patterns have the form $S B B \ldots B S$, where there are $k_{1}>0$ small spikes $S$ and $k_{2}=k-k_{1}>0$ large spikes $B$ arranged in any order across the interval. Neglecting the positioning of large and small spikes in a spike sequence, we show in Principal Result 4.3 that for $k>1$ there are $k-1$ asymmetric $k$-spike equilibrium patterns that bifurcate from the symmetric $k$-spike solution branch at precisely the threshold value $\mathcal{A}=\mathcal{A}_{k S}$ where $k-1$ small eigenvalues for the symmetric $k$-spike solution branch simultaneously cross through zero. The $k-1$ bifurcating branches correspond to the number of small spikes $S$ in a spike sequence $S B B \ldots B S$.

For $D=0.75$, in Fig. 1(a) we plot a bifurcation diagram of the norm of $v$ versus $\mathcal{A}$ for the symmetric $k$-spike solution branches for $k=1, \ldots, 4$. The dotted curves in Fig. 1(a) show the asymmetric $k$-spike patterns that bifurcate off of the symmetric $k$-spike solution branch at $\mathcal{A}=\mathcal{A}_{k S}$. In Fig. 1(b) we illustrate the stability properties of the symmetric solution branches when $\tau$ is below the Hopf bifurcation threshold $\tau_{h L}$. In this figure, we show the range in $\mathcal{A}$ where the pattern is stable with respect to the large eigenvalues but not the small eigenvalues. In Fig. 2(a) we plot a symmetric 


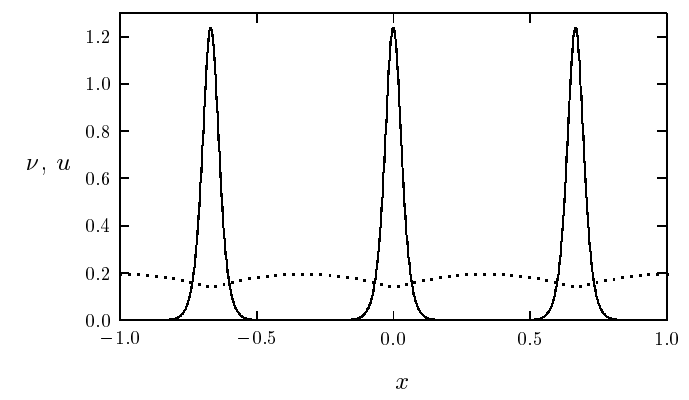

(a) Symmetric three-spike pattern

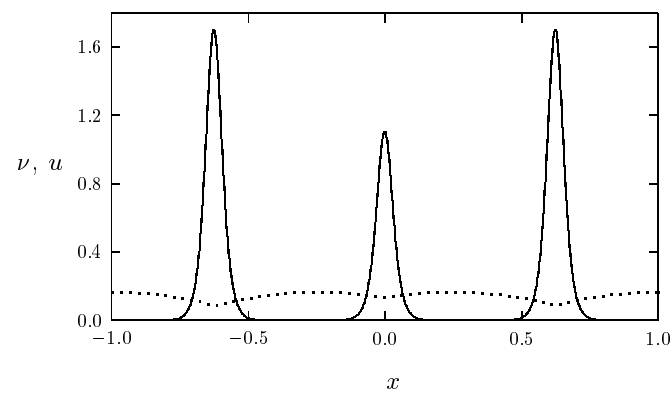

(b) Asymmetric three-spike pattern

FIG. 2. Left figure: symmetric three-spike solution on the upper branch of the bifurcation diagram when $D=0.75, \mathcal{A}=9.0$, and $\varepsilon=0.02$. Right figure: a three-spike BSB asymmetric pattern with $D=0.75, \mathcal{A}=10.5$, and $\varepsilon=0.02$. In these plots, $v$ are the solid curves and $u$ are the dotted curves.

three-spike solution at a particular point on the upper branch. An asymmetric three-spike solution of the form BSB is shown in Fig. 2(b),

Similar analyses of the existence and stability of $k$-spike patterns have been previously performed for the Gierer-Meinhardt (GM) model (cf. [13]), widely used to model localization in biological pattern formation (cf. [26]). This system can be written in dimensionless form as (cf. [17])

$a_{t}=\varepsilon^{2} a_{x x}-a+\frac{a^{p}}{h^{q}}, \quad \tau h_{t}=D h_{x x}-h+\varepsilon^{-1} \frac{a^{m}}{h^{s}}, \quad|x|<1 ; \quad a_{x}( \pm 1, t)=h_{x}( \pm 1, t)=0$.

Here $0<\varepsilon^{2} \ll 1, D>0$, and $\tau \geqslant 0$ are constants. The GM exponents $(p, q, m, s)$ are assumed to satisfy

$$
p>1, \quad q>0, \quad m>1, \quad s \geqslant 0, \quad \text { with } \quad \zeta \equiv \frac{q m}{(p-1)}-(s+1)>0 .
$$

The stability of symmetric $k$-spike equilibrium solutions to (1.4) was analyzed in [17] for the case $\tau=0$ and in [46] for $\tau>0$. Asymmetric $k$-spike equilibria were constructed in [45] and [10], and a partial stability analysis for asymmetric patterns was given in [45]. In [47] asymmetric spike patterns for the GM model in two spatial dimensions were analyzed rigorously. However, the rigorous proofs given in [47] do not directly carry over to the one-dimensional situation for the GS model studied here. The stability of a one-spike solution to (1.4) on the infinite line was studied in [9], and the dynamics of spikes was studied in [16], [42], and [43]. For the GM model, the relationship between translational instabilities of symmetric $k$-spike patterns and the emergence of asymmetric spike patterns is emphasized in [45] and [47]. The results in this study show that there is a rather close spectral equivalence between translational instabilities in the GM model and the GS model in the low feed-rate regime.

The method that we use to study translational instabilities for the GS model is related to the SLEP (singular limit eigenvalue problem) method developed and applied in [32]-[34], in the context of analyzing the translational stability of hyperbolic-tangent type interface solutions to singularly perturbed two-component systems in the semi-strong interaction limit. For these class of systems, where the nonlinearity associated with the fast variable is bistable, each transition layer is closely approximated by a heteroclinic connection. The resulting spectral problem has only small eigenvalues, also called critical spectra, that tend to zero linearly with the thickness $\varepsilon$ of the interface. 
Under certain reasonable hypotheses, it was proved in [32] that a one-layer solution is stable. Under the same conditions, the stability of an $n$-layer solution was proved in [33] by first reducing the spectral problem to a certain matrix eigenvalue problem. Similar problems involving only critical spectra occur in certain phase separation models with an associated variational principle including the Cahn-Hilliard, Allen-Cahn, and phase-field models (cf. [1]-[4]), and the analysis of lamellar states for Diblock copolymers (cf. [40]). The stability problem for these transition layer structures is in marked contrast to the study of the stability of homoclinic-type spike patterns for the GS or GM models where there is no variational structure, and where there are both large eigenvalues, with $\lambda=O(1)$, and small eigenvalues in the spectrum of the linearization.

In $\S 5$ we investigate translational instabilities for the GS model in the intermediate regime when $\tau$ is asymptotically large as $\varepsilon \rightarrow 0$. For a $k$-spike equilibrium solution in this regime it was shown in [7] and [21] that the NLEP governing instabilities on an $O(1)$ time-scale has a Hopf bifurcation when $\tau=O\left(\mathcal{A}^{4}\right) \gg 1$ (see Principal Result A.3 below). Therefore, in the intermediate regime, this suggests that for some asymptotic range where $\tau \gg 1$ an instability with respect to the small eigenvalues may be possible before the onset of the Hopf bifurcation in the spike profile. This exchange in the dominant instability mechanism as $\tau$ is increased, which has no known counterpart in the GM model [1.4], was suggested in [28] and [29] in the context of a one-spike solution for the infinite-line problem. For a one-spike equilibrium solution on a finite interval, we use a formal asymptotic analysis to obtain an auxiliary problem in Principal Result 5.1 that the small eigenvalue satisfies. By studying this problem rigorously, in Proposition 5.2 we show that as $\tau$ increases past some critical value there is a drift instability due to a Hopf bifurcation, which leads to small-scale oscillations in the spike-layer location. As $\tau$ is increased even further, a pair of unstable complex conjugate eigenvalues merge onto the positive real axis, which results in a slow monotonic drift of the spike layer location. The critical value for the onset of an oscillatory drift instability occurs on the asymptotic range $\tau=O\left(\mathcal{A}^{-2} \varepsilon^{-2}\right)$. A related type of Hopf bifurcation, followed by a monotonic drift instability as a reaction-time constant is increased, has been analyzed in [15] and [27] for hyperbolic tangent-type interfaces associated with a two-component reaction-diffusion system with bistable nonlinearities. Alternatively, for a three-component reaction-diffusion system it was shown numerically in [35] that the Hopf bifurcation occurs after the onset of a monotonic drift instability as a reaction-time parameter is increased.

In $§ 6$ we make a few remarks and suggest a few open problems for further study.

Finally, we remark on the mathematical rigor of our approach. The key results of formal asymptotic derivations are labeled as Principal Results. Rigorous results based either on exact calculations or mathematical theory are labeled as Propositions. More specifically, in $\S 3$ we use a formal asymptotic analysis to construct equilibrium $k$-spike solutions and to derive the auxiliary problem in Principal Result 3.1 for the small eigenvalues. The results in Propositions 3.2-3.6 follow from exact calculations involving this problem. The construction of asymmetric patterns in $\S 4$ is done using formal asymptotics. In $\$ 5$ we present a formal derivation of the auxiliary problem for drift instabilities leading to Principal Result 5.1. The existence of a drift instability threshold in Proposition 5.2 follows from a rigorous analysis of this problem.

\section{Symmetric $k$-spike equilibria: the stability problem}

In this section and in Appendix A we summarize some previous results for the existence and stability, on an $O(1)$ time-scale, of symmetric $k$-spike patterns to the GS model $(1.3)$ in the low feed-rate regime $\mathcal{A}=O(1)$ and in the intermediate regime $O(1) \ll \mathcal{A} \ll O\left(\varepsilon^{-1 / 2}\right)$. In the low 
feed-rate regime, a symmetric $k$-spike equilibrium solution to (1.3) was constructed in $\S 2$ of [21] using the method of matched asymptotic expansions. We now briefly outline this derivation, as many of the formulae are needed below in $\S 3-\S 5$.

For a symmetric spike pattern the spikes have equal height so that $u\left(x_{j}\right)=U$ for $j=1, \ldots, k$, where

$$
x_{j}=-1+\frac{2 j-1}{k}, \quad j=1, \ldots, k .
$$

In the inner region near the $j^{\text {th }}$ spike, we let $y=\varepsilon^{-1}\left(x-x_{j}\right)$. In each inner region, we find that $u \sim U+O(\varepsilon)$. Therefore, from 1.3a), the leading-order inner solution for $v$ is $v \sim w /(\mathcal{A} U)$, where $w(y)=\frac{3}{2} \operatorname{sech}^{2}(y / 2)$ is the homoclinic solution to

$$
w^{\prime \prime}-w+w^{2}=0, \quad-\infty<y<\infty ; \quad w \rightarrow 0 \quad \text { as }|y| \rightarrow \infty, \quad w^{\prime}(0)=0, \quad w(0)>0 .
$$

In the outer region, defined away from an $O(\varepsilon)$ region near each spike, $v$ is exponentially small and the term $\varepsilon^{-1} u v^{2}$ in $1.3 \mathrm{~b}$ can be approximated by a Dirac mass. Thus, the outer solution for $u$ satisfies

$$
D u_{x x}+(1-u)-\frac{6}{\mathcal{A}^{2} U} \sum_{j=1}^{k} \delta\left(x-x_{j}\right)=0, \quad-1<x<1 ; \quad u_{x}( \pm 1)=0 .
$$

In obtaining 2.3 , we used $\int_{-\infty}^{\infty} w^{2} \mathrm{~d} y=6$. The solution to 2.3 is

$$
u(x)=1-\frac{6}{\mathcal{A}^{2} U} \sum_{j=1}^{k} G\left(x ; x_{j}\right)
$$

where $G\left(x ; x_{j}\right)$ is the Green's function, satisfying

$$
D G_{x x}-G=-\delta\left(x-x_{j}\right), \quad-1<x<1 ; \quad G_{x}\left( \pm 1 ; x_{j}\right)=0 .
$$

We define $a_{g} \equiv \sum_{i=1}^{k} G\left(x_{j} ; x_{i}\right)$, where the spike locations satisfy 2.1). From an explicit calculation of $G\left(x ; x_{j}\right)$, we deduce that $a_{g}$ is independent of $j$, and is given by

$$
a_{g} \equiv \sum_{i=1}^{k} G\left(x_{j} ; x_{i}\right)=\left[2 \sqrt{D} \tanh \left(\theta_{0} / k\right)\right]^{-1} .
$$

Evaluating 2.4 at $x=x_{j}$, where $u\left(x_{j}\right)=U$, we obtain a quadratic equation for $U$,

$$
U(U-1)=-\frac{6 a_{g}}{\mathcal{A}^{2}} .
$$

In this way, the following formal result for symmetric $k$-spike equilibrium solutions to (1.3) was obtained in Principal Result 2.1 of [21]:

PRincipal ReSUlt 2.1 (from [21]) Let $\varepsilon \rightarrow 0$, with $\mathcal{A}=O(1)$ and $D=O(1)$ in $[1.3$ ). Then, when $\mathcal{A}>\mathcal{A}_{k e}$, there are two symmetric $k$-spike equilibrium solutions to 1.3 given asymptotically by

$$
\nu_{ \pm}(x) \sim \frac{1}{\mathcal{A} U_{ \pm}} \sum_{j=1}^{k} w\left[\varepsilon^{-1}\left(x-x_{j}\right)\right], \quad u_{ \pm}(x) \sim 1-\frac{1-U_{ \pm}}{a_{g}} \sum_{j=1}^{k} G\left(x ; x_{j}\right)
$$


We refer to $u_{+}, v_{+}$and $u_{-}, v_{-}$as the small and large solution, respectively. In $(2.8), w$ and $G$ satisfy (2.2) and (2.5), respectively. In addition, $U_{ \pm}$are the roots of (2.7) given by

$$
U_{ \pm}=\frac{1}{2}\left[1 \pm \sqrt{1-\frac{\mathcal{A}_{k e}^{2}}{\mathcal{A}^{2}}}\right], \quad \mathcal{A}_{k e} \equiv \sqrt{\frac{12 \theta_{0}}{\tanh \left(\theta_{0} / k\right)}}, \quad \theta_{0} \equiv D^{-1 / 2} .
$$

Although we have only sketched the formal derivation of equilibrium solutions in the low feedrate regime, there are several methods that can be used to construct these solutions rigorously. In [11] a rigorous geometric singular perturbation approach was used to establish the existence of a one-spike solution and a periodic solution to a different dimensionless form of the infinite-line GS model (cf. Theorem 4.3 of [11]). For a one-spike solution on the infinite line, the threshold $\mathcal{A}_{1 e}^{\infty}=\sqrt{12} D^{-1 / 4}$ for the low feed-rate regime is equivalent to that given in Theorem 4.3 of [11]. This infinite-line threshold can be recovered by setting $k=1$ and then letting $D \rightarrow 0$ in the formula for $\mathcal{A}_{k e}$ in 2.9 .

In Fig. 1(a) we plot the bifurcation diagram of the $L_{2}$ norm $|v|_{2}$ versus $\mathcal{A}$ for $D=0.75$, and $k=1, \ldots, 4$. These are the solid curves in Fig. 1(a) Using (2.8) and (2.9), we obtain

$$
|v|_{2} \equiv\left(\varepsilon^{-1} \int_{-1}^{1} v^{2} \mathrm{~d} x\right)^{1 / 2} \sim \frac{2 \sqrt{6 k}}{\mathcal{A}}\left[1 \pm \sqrt{1-\frac{\mathcal{A}_{k e}^{2}}{\mathcal{A}^{2}}}\right]^{-1} .
$$

The existence thresholds $\mathcal{A}_{k e}$ are the saddle-node points in Fig. 1(a) For the values $\mathcal{A}=9.0$, $D=0.75$, and $\varepsilon=0.02$, a symmetric three-spike solution is shown in Fig. 2(a) The classification of small and large solution refers to low and high concentrations of $v$ in the core of the spike. Smaller concentrations of $u$ in the core of the spike generate larger amplitudes for $v$. Hence, each upper branch (upper solid curve) in Fig. 1(a) corresponds to the large solution, while each lower branch corresponds to the small solution. A convenient way to parametrize these solution branches is to introduce a parameter $s$ defined by $s \equiv\left(1-U_{ \pm}\right) / U_{ \pm}$. Then, from [2.9), we get

$$
\mathcal{A}=\mathcal{A}_{k e} \frac{1+s}{2 \sqrt{s}}, \quad s=\frac{1-U_{ \pm}}{U_{ \pm}}, \quad 0<s<\infty
$$

The large solution $u_{-}, v_{-}$corresponds to the range $1<s<\infty$, while the small solution $u_{+}, v_{+}$ corresponds to $0<s<1$. The existence threshold $\mathcal{A}_{k e}$ is at $s=1$.

To analyze the stability of symmetric $k$-spike equilibrium solutions we let

$$
u(x, t)=u_{ \pm}(x)+e^{\lambda t} \eta(x), \quad v(x, t)=v_{ \pm}(x)+e^{\lambda t} \phi(x),
$$

where $\eta \ll 1$ and $\phi \ll 1$. Substituting 2.12$]$ into $(1.3)$, and linearizing, we obtain the eigenvalue problem

$$
\begin{array}{rlrl}
\varepsilon^{2} \phi_{x x}-\phi+2 \mathcal{A} u_{ \pm} \nu_{ \pm} \phi+\mathcal{A} \eta v_{ \pm}^{2} & =\lambda \phi, & -1<x<1 ; & \phi_{x}( \pm 1)=0, \\
D \eta_{x x}-\eta-\varepsilon^{-1} v_{ \pm}^{2} \eta-2 \varepsilon^{-1} u_{ \pm} \nu_{ \pm} \phi=\tau \lambda \eta, & -1<x<1 ; & \eta_{x}( \pm 1)=0 .
\end{array}
$$

There are two classes of eigenvalues and eigenfunctions of 2.13); the large eigenvalues for which $\lambda=O(1)$ as $\varepsilon \rightarrow 0$, and the small eigenvalues for which $\lambda=O\left(\varepsilon^{2}\right)$ as $\varepsilon \rightarrow 0$. 
For the large eigenvalues with $\lambda=O(1)$ as $\varepsilon \rightarrow 0$, which determine the stability of a $k$-spike equilibrium solution on an $O(1)$ time-scale, the corresponding eigenfunction has the form

$$
\phi(x) \sim \sum_{j=1}^{k} b_{j} \Phi\left[\varepsilon^{-1}\left(x-x_{j}\right)\right],
$$

where $\int_{-\infty}^{\infty} w(y) \Phi(y) \mathrm{d} y \neq 0$. Here the coefficients $b_{j}$, for $j=1, \ldots, k$, are found to be related to the eigenvectors of a certain matrix eigenvalue problem. This stability problem, which involves the analysis of a nonlocal eigenvalue problem, was studied in [7], [8], [21], and [29]. Spectral results for this problem in the low feed-rate and intermediate regimes of the GS model (1.3) are summarized in Appendix A.

In this paper we study the small eigenvalues of 2.13 that lead to slow translational instabilities. In contrast to 2.14, for $\varepsilon \rightarrow 0$ the corresponding eigenfunction in $\S 3$ has the form

$$
\phi(x) \sim \sum_{j=1}^{k}\left(c_{j} w^{\prime}\left[\varepsilon^{-1}\left(x-x_{j}\right)\right]+\varepsilon c_{j} \phi_{1 j}\left[\varepsilon^{-1}\left(x-x_{j}\right)\right]\right) .
$$

The leading term in (2.15) is simply the translation mode associated with the spike profile $w$. The correction term $\phi_{1 j}$ in (2.15) is shown below in (3.11) (see also (3.27) of Proposition 3.2) to be proportional to the spike profile $w\left[\varepsilon^{-1}\left(x-x_{j}\right)\right]$ of the $j^{\text {th }}$ spike. In $\$ 3$ we will derive explicit formulae for the coefficients $c_{j}$ in terms of a matrix eigenvalue problem, and we will calculate the small eigenvalues $\lambda_{j}=O\left(\varepsilon^{2}\right)$ precisely.

Our method to analyze translational instabilities is related to the SLEP method, originally developed and analyzed rigorously for the study of transition-layer stability associated with twocomponent reaction-diffusion systems in the semi-strong limit (cf. [32]-[34]). In these systems, the nonlinearity in the fast variable is of bistable type. In these works, the study of the critical spectrum is also reduced to the study of the spectrum of a matrix eigenvalue problem. However, since the leading term in 2.15 is a monopole when the profile $w$ is a transition layer, only the first term in (2.15) is sufficient for calculating the spectrum. For the transition layer case, the critical spectra are $O(\varepsilon)$ as $\varepsilon \rightarrow 0$, and the matrix manipulations required to calculate the critical spectrum are relatively straightforward. In addition, it was shown in [33], under reasonable hypotheses, that the critical spectra are all negative for an $n$-layer solution. Therefore, such a solution is stable and there are no bifurcations to other patterns. Alternatively, in our analysis of spike stability, since the leading term in $(2.15)$ is a dipole and the $O(\varepsilon)$ term in (2.15) is found to be a monopole, these two terms have the same effective order as $\varepsilon \rightarrow 0$ in our analysis of the critical spectrum of $O\left(\varepsilon^{2}\right)$ for 2.13. In addition, in contrast to the critical spectrum for transition layers, the critical spectrum for spikes in the GS model can have bifurcations.

\section{Slow translational instabilities for a symmetric $k$-spike pattern}

For $\varepsilon \ll 1$, we now study the stability of the symmetric $k$-spike equilibrium solutions of Principal Result 2.1 with respect to the small eigenvalues of 2.13 of order $O\left(\varepsilon^{2}\right)$. The first part of the analysis is to reduce (2.13) to the study of a matrix eigenvalue problem. We begin by writing 2.13 in the form

$$
\begin{aligned}
& L_{\varepsilon} \phi+\mathcal{A} \eta v_{ \pm}^{2}=\lambda \phi, \quad-1<x<1 ; \quad \phi_{x}( \pm 1)=0, \\
& D \eta_{x x}-\left(1+\tau \lambda+\varepsilon^{-1} \nu_{ \pm}^{2}\right) \eta=2 \varepsilon^{-1} u_{ \pm} \nu_{ \pm} \phi, \quad-1<x<1 ; \quad \eta_{x}( \pm 1)=0,
\end{aligned}
$$


where $u_{ \pm}$and $v_{ \pm}$are given in (2.8) of Principal Result 2.1. Here the operator $L_{\varepsilon}$ is defined by

$$
L_{\varepsilon} \phi \equiv \varepsilon^{2} \phi_{x x}-\phi+2 \mathcal{A} u_{ \pm} v_{ \pm} \phi
$$

Since $\lambda=O\left(\varepsilon^{2}\right),\left(3.1 \mathrm{~b}\right.$ yields $\tau \lambda \ll 1$ unless $\tau=O\left(\varepsilon^{-2}\right)$. In the derivation below, we will assume that $\tau=O(1)$ so that the small eigenvalues are asymptotically independent of $\tau$. It is important to emphasize here that we cannot naively replace $L_{\varepsilon}$ by the noninvertible Fisher operator $L_{0}$ of the NLEP problem of Principal Result A.1. Although the coefficients in these two operators are $O(\varepsilon)$ close near a spike, the small spatial variations in the coefficients of $L_{\varepsilon}$ are important for estimating the small eigenvalues for $\varepsilon \ll 1$.

Since $v_{ \pm}$is localized near each spike $x_{j}$, the spike pattern is nearly translationally invariant. To show this, we differentiate the equilibrium problem for $1.3 \mathrm{a}$ with respect to $x_{1}$ to get

$$
L_{\varepsilon} v_{ \pm x}=-\mathcal{A} u_{ \pm x} v_{ \pm}^{2} .
$$

Defining $w_{j}=w_{j}\left(y_{j}\right) \equiv w\left[\varepsilon^{-1}\left(x-x_{j}\right)\right]$, where $w$ satisfies 2.2, we calculate from 2.8) that for $x$ near $x_{j}$,

$$
v_{ \pm} \sim \frac{1}{\mathcal{A} U_{ \pm}} w_{j}, \quad v_{ \pm x} \sim \frac{\varepsilon^{-1}}{\mathcal{A} U_{ \pm}} w_{j}^{\prime}
$$

Substituting (3.3) into 3.2), for $x$ near $x_{j}$ we obtain

$$
L_{\varepsilon} w_{j}^{\prime} \sim-\varepsilon U_{ \pm}^{-1} u_{ \pm x} w_{j}^{2}=O(\varepsilon)
$$

This suggests that we look for an eigenfunction to 3.1 in the form

$$
\phi=\phi_{0}+\varepsilon \phi_{1}+\cdots, \quad \eta(x)=\varepsilon \eta_{0}(x)+\cdots,
$$

where, for some coefficients $c_{j}$, with $j=1, \ldots, k$, to be determined, we have

$$
\phi_{0} \equiv \sum_{j=1}^{k} c_{j} w_{j}^{\prime}\left[\varepsilon^{-1}\left(x-x_{j}\right)\right], \quad \phi_{1} \equiv \sum_{j=1}^{k} c_{j} \phi_{1 j}\left[\varepsilon^{-1}\left(x-x_{j}\right)\right] .
$$

Here and below we have defined $\langle\zeta\rangle_{j} \equiv\left(\zeta\left(x_{j+}\right)+\zeta\left(x_{j-}\right)\right) / 2$ and $[\zeta]_{j} \equiv \zeta\left(x_{j+}\right)-\zeta\left(x_{j-}\right)$, where $\zeta\left(x_{j \pm}\right)$ are the one-sided limits of $\zeta(x)$ as $x \rightarrow x_{j \pm}$. In particular, by differentiating the outer solution $u_{ \pm}$given in (2.8), it follows that the equilibrium positions for $x_{j}$ of (2.1) satisfy

$$
\left\langle u_{ \pm x}\right\rangle_{j}=0, \quad j=1, \ldots, k .
$$

We substitute 3.5a into 3.1a) with $\lambda=O\left(\varepsilon^{2}\right) \ll 1$, and we use 3.4. For $x$ near $x_{j}$, we find that $\phi_{1 j}(y)$ satisfies

$$
c_{j} L_{\varepsilon} \phi_{1 j} \sim f\left(x_{j}+\varepsilon y_{j}\right) w_{j}^{2}, \quad f(x) \equiv \frac{c_{j} u_{ \pm x}(x)}{U_{ \pm}}-\frac{\eta_{0}(x)}{\mathcal{A} U_{ \pm}^{2}} .
$$

Substituting 3.5a) into 3.1b), we deduce that $\eta_{0}$ satisfies

$$
D \eta_{0 x x}-\left(1+\varepsilon^{-1} v_{ \pm}^{2}\right) \eta_{0} \sim 2 \varepsilon^{-2} u_{ \pm} v_{ \pm}\left(\phi_{0}+\varepsilon \phi_{1}\right), \quad-1<x<1 ; \quad \eta_{0 x}( \pm 1)=0 .
$$


Since $\phi_{0}$ is proportional to the odd function $w_{j}^{\prime}$ near $x=x_{j}$, the term multiplied by $\phi_{0}$ in 3.8 , behaves like a dipole near $x=x_{j}$. Hence, for $\varepsilon \ll 1$ and $x$ near $x_{j}$, this term is proportional to $\delta^{\prime}\left(x-x_{j}\right)$ for $j=1, \ldots, k$, where $\delta(x)$ is the delta function. Thus, $\eta_{0}$ is discontinuous across $x=x_{j}$.

Since $\eta_{0}$ is discontinuous across $x=x_{j}$, it would suggest that $f(x)$ in 3.7 is also discontinuous across $x=x_{j}$. However, this is not the case, as we find that the first term on the right-hand side of $f(x)$ in $(3.7)$ exactly cancels this singularity. To see this, we differentiate the equilibrium problem for $u$ in $1.3 \mathrm{~b}$ with respect to $x$, and subtract appropriate multiples of the resulting equation and (3.8) to find that the dipole term proportional to $\phi_{0}$ cancels exactly. In this way, we find for $x$ near $x_{j}$ that $f(x)$ satisfies

$D f_{x x}-\left(1+\varepsilon^{-1} \nu_{ \pm}^{2}\right) f=-\frac{2 \varepsilon^{-1} c_{j}}{\mathcal{A} U_{ \pm}^{2}} \phi_{1 j} u_{ \pm} \nu_{ \pm}, \quad-1<x<1 ; \quad f_{x}( \pm 1)=\frac{c_{j} u_{ \pm x x}( \pm 1)}{U_{ \pm}}$

Assume for the moment that $\phi_{1 j}$ is an even function. Then the right-hand side of 3.9 is a monopole term. Therefore, we would conclude that $f(x)$ is continuous across $x=x_{j}$, and consequently $\langle f\rangle_{j}=f\left(x_{j}\right)$. Since $\left\langle u_{ \pm x}\right\rangle_{j}=0$ from 3.6, 3.7) reduces for $\varepsilon \ll 1$ to

$$
c_{j} L_{\varepsilon} \phi_{1 j} \sim f\left(x_{j}\right) w_{j}^{2}, \quad f\left(x_{j}\right)=-\frac{\left\langle\eta_{0}\right\rangle_{j}}{\mathcal{A} U_{ \pm}^{2}}
$$

Since $L_{\varepsilon} w_{j}=w_{j}^{2}+O(\varepsilon)$, and $L_{\varepsilon}$ is uniformly invertible on function spaces of even functions, we can solve 3.10 uniquely as

$$
c_{j} \phi_{1 j} \sim f\left(x_{j}\right) w_{j} .
$$

Since $w_{j}$ is even, so is $\phi_{1 j}$, and the calculation is self-consistent. Alternatively, if we had initially assumed that $\phi_{1 j}$ was odd in 3.9 we would reach a contradiction. If $\phi_{1 j}$ is an odd function, then the right-hand side of (3.9) is a dipole term and for $\varepsilon \rightarrow 0$ can be represented as $\varepsilon d \delta^{\prime}\left(x-x_{j}\right)$ for some $O$ (1) constant $d$. Therefore, for $\varepsilon \ll 1$, the leading order behavior of $f(x)$ is still continuous across $x=x_{j}$. Hence, 3.10 still holds, and the resulting form 3.11 contradicts the assumed oddness of $\phi_{1 j}$.

This discussion shows that the term in (3.8) proportional to $\phi_{1}$ behaves like a linear combination of $\delta\left(x-x_{j}\right)$. Most importantly, this shows in 3.12) below that the effect of the monopole term for $\phi_{1}$ is of the same order in $\varepsilon$ as the dipole term proportional to $\phi_{0}$. Therefore, to calculate an eigenvalue of order $O\left(\varepsilon^{2}\right)$, we need to determine the asymptotic eigenfunction for $\phi$ in 3.5a to both the $O(1)$ and $O(\varepsilon)$ terms.

Next, in the sense of distributions, we calculate the strengths of the dipole and monopole terms appearing on the right-hand side of (3.8). Using (3.3) for $v_{ \pm}$and $u_{ \pm} \sim U_{ \pm}$for $x$ near $x_{j}$, we calculate from 3.5b), 3.10, and 3.11) that, for $x$ near $x_{j}$,

$$
\begin{aligned}
& 2 \varepsilon^{-2} u_{ \pm} v_{ \pm} \phi_{0} \rightarrow \frac{6 c_{j}}{\mathcal{A}} \delta^{\prime}\left(x-x_{j}\right), \quad \varepsilon^{-1} v_{ \pm}^{2} \rightarrow \frac{6}{\mathcal{A}^{2} U_{ \pm}^{2}} \delta\left(x-x_{j}\right), \\
& 2 \varepsilon^{-1} u_{ \pm} v_{ \pm} \phi_{1} \rightarrow \frac{12 f\left(x_{j}\right)}{\mathcal{A}} \delta\left(x-x_{j}\right)=-\frac{12\left\langle\eta_{0}\right\rangle_{j}}{\mathcal{A}^{2} U_{ \pm}^{2}} \delta\left(x-x_{j}\right) .
\end{aligned}
$$


Here we have used $\int_{-\infty}^{\infty} w_{j}^{2} \mathrm{~d} y=6$. Substituting 3.12 into 3.8 , we see that $\eta_{0}$ satisfies

$D \eta_{0 x x}-\left[1+\frac{6}{\mathcal{A}^{2} U_{ \pm}^{2}} \sum_{j=1}^{k} \delta\left(x-x_{j}\right)\right] \eta_{0}=\frac{6}{\mathcal{A}} \sum_{j=1}^{k} c_{j} \delta^{\prime}\left(x-x_{j}\right)-\frac{12}{\mathcal{A}^{2} U_{ \pm}^{2}} \sum_{j=1}^{k}\left\langle\eta_{0}\right\rangle_{j} \delta\left(x-x_{j}\right)$,

with $\eta_{0 x}( \pm 1)=0$. Using (2.7) for $U_{ \pm}$, and the definition of $s$ in 2.11), we can write

$$
\frac{6}{\mathcal{A}^{2} U_{ \pm}^{2}}=\frac{s}{a_{g}}, \quad s=\frac{1-U_{ \pm}}{U_{ \pm}},
$$

where $a_{g}$ is given in 2.6. Substituting (3.14) into 3.13), we conclude that 3.13 is equivalent to

$$
\begin{gathered}
D \eta_{0 x x}-\eta_{0}=0, \quad-1<x<1 ; \quad \eta_{0 x}( \pm 1)=0, \\
{\left[D \eta_{0}\right]_{j}=\frac{6 c_{j}}{\mathcal{A}} ; \quad\left[D \eta_{0 x}\right]_{j}=\frac{-s}{a_{g}}\left\langle\eta_{0}\right\rangle_{j}, \quad j=1, \ldots, k .}
\end{gathered}
$$

Next, we estimate the small eigenvalue. We substitute $(3.5)$ into $3.1 \mathrm{a}$ and then multiply both sides of $3.1 \mathrm{a}$ by $w_{j}^{\prime}$. By integrating the resulting equation across the domain, we get

$$
\sum_{i=1}^{k}\left(w_{j}^{\prime}, c_{i} L_{\varepsilon} w_{i}^{\prime}\right)+\varepsilon \mathcal{A}\left(\eta_{0} v_{ \pm}^{2}, w_{j}^{\prime}\right)+\varepsilon \sum_{i=1}^{k}\left(w_{j}^{\prime}, c_{i} L_{\varepsilon} \phi_{1 i}\right) \sim \lambda \sum_{i=1}^{k} c_{i}\left(w_{j}^{\prime}, w_{i}^{\prime}\right) .
$$

Here we have defined $(f, g) \equiv \int_{-1}^{1} f(x) g(x) \mathrm{d} x$. Since $w_{j}^{\prime}$ is exponentially small away from $x=x_{j}$, it follows that to within negligible exponentially small terms the dominant contribution to the sums in 3.16 arises from $i=j$. Thus, 3.16 reduces asymptotically to

$$
c_{j}\left(w_{j}^{\prime}, L_{\varepsilon} w_{j}^{\prime}\right)+\varepsilon \mathcal{A}\left(\eta_{0} v_{ \pm}^{2}, w_{j}^{\prime}\right)+\varepsilon\left(w_{j}^{\prime}, c_{j} L_{\varepsilon} \phi_{1 j}\right) \sim \lambda c_{j}\left(w_{j}^{\prime}, w_{j}^{\prime}\right) .
$$

We then integrate the third term on the left-hand side of 3.17 by parts. Since $L_{\varepsilon}$ is self-adjoint, we can then use 3.4 for $L_{\varepsilon} w_{j}^{\prime}$ and 2.8 for $v_{ \pm}$. Since the integrands are localized near $x=x_{j}$, we can write the resulting integrals in terms of $y=\varepsilon^{-1}\left(x-x_{j}\right)$ to get

$$
\begin{array}{r}
-\frac{\varepsilon^{2} c_{j}}{U_{ \pm}} \int_{-\infty}^{\infty} w^{\prime} w^{2} u_{ \pm x} \mathrm{~d} y+\frac{\varepsilon^{2}}{\mathcal{A} U_{ \pm}^{2}} \int_{-\infty}^{\infty} w^{2} w^{\prime} \eta_{0} \mathrm{~d} y-\frac{\varepsilon^{3} c_{j}}{U_{ \pm}} \int_{-\infty}^{\infty} \phi_{1 j} w^{2} u_{ \pm x} \mathrm{~d} y \\
\sim \varepsilon \lambda c_{j}\left(\int_{-\infty}^{\infty} w^{\prime 2} \mathrm{~d} y\right)
\end{array}
$$

where $w$ satisfies 2.2 . In this expression we have labeled $\eta_{0} \equiv \eta_{0}\left(x_{j}+\varepsilon y\right)$ and $u_{ \pm x} \equiv u_{ \pm x}\left(x_{j}+\varepsilon y\right)$.

Using (3.11) for $\phi_{1 j}$, and 3.7) for $f(x)$, we can write 3.18 more compactly as

$$
-\varepsilon^{2} \int_{-\infty}^{\infty} w^{\prime} w^{2} f\left(x_{j}+\varepsilon y\right) \mathrm{d} y-\frac{\varepsilon^{3} f\left(x_{j}\right)}{U_{ \pm}} \int_{-\infty}^{\infty} w^{3} u_{ \pm x}\left(x_{j}+\varepsilon y\right) \mathrm{d} y \sim \varepsilon \lambda c_{j}\left(\int_{-\infty}^{\infty} w^{\prime 2} \mathrm{~d} y\right) .
$$

The second integral on the left-hand side of 3.19 is $O\left(\varepsilon^{4}\right)$ since $w$ is even and $\left\langle u_{ \pm x}\right\rangle_{j}=0$ from (3.6.). 
From (3.9) and 3.11), it follows that $f(x)$ is continuous but not differentiable across $x=x_{j}$. Therefore, we must calculate the first term on the left-hand side of 3.19 by first expanding $f\left(x_{j}+\varepsilon y\right)$ in one-sided Taylor series. After doing so, we then integrate the resulting equation by parts. This yields

$$
\int_{-\infty}^{\infty} w^{\prime} w^{2} f\left(x_{j}+\varepsilon y\right) \mathrm{d} y \sim-\frac{\varepsilon}{3}\left\langle f^{\prime}\right\rangle_{j} \int_{-\infty}^{\infty} w^{3} \mathrm{~d} y .
$$

Using (3.7) for $f(x)$, and $\left\langle u_{ \pm x x}\right\rangle_{j}=\left(U_{ \pm}-1\right) / D$ from 2.3, we calculate

$$
\left\langle f^{\prime}\right\rangle_{j}=\frac{c_{j}\left(U_{ \pm}-1\right)}{U_{ \pm} D}-\frac{\left\langle\eta_{0 x}\right\rangle_{j}}{\mathcal{A} U_{ \pm}^{2}} .
$$

Then we substitute 3.21) and 3.20 into 3.19, to obtain

$$
\lambda c_{j} \sim \frac{\varepsilon^{2}}{3}\left[\frac{c_{j}\left(U_{ \pm}-1\right)}{U_{ \pm} D}-\frac{\left\langle\eta_{0 x}\right\rangle_{j}}{\mathcal{A} U_{ \pm}^{2}}\right]\left(\frac{\int_{-\infty}^{\infty} w^{3} \mathrm{~d} y}{\int_{-\infty}^{\infty} w^{\prime 2} \mathrm{~d} y}\right) .
$$

Here $\left\langle\eta_{0 x}\right\rangle_{j}$ is to be found from 3.15. Finally, we write 3.22 and 3.15 in terms of $\tilde{\eta}_{0}$ defined by

$$
\eta_{0} \equiv-\frac{6 a_{g}}{\mathcal{A}} \tilde{\eta}_{0}
$$

In addition, we calculate the ratio of the two integrals in 3.22 using $w(y)=\frac{3}{2} \operatorname{sech}^{2}(y / 2)$. In this way, we formally obtain the following problem that determines the small eigenvalues:

PRINCIPAL RESUlt 3.1 Let $\varepsilon \ll 1$ and $\tau=O(1)$. Then the eigenvalues of 2.13 of order $O\left(\varepsilon^{2}\right)$ satisfy

$$
\lambda c_{j} \sim 2 \varepsilon^{2} s\left[\left\langle\tilde{\eta}_{0 x}\right\rangle_{j}-\frac{c_{j}}{D}\right], \quad j=1, \ldots, k,
$$

where $\left\langle\tilde{\eta}_{0 x}\right\rangle_{j}$ is obtained from the solution to the following auxiliary problem:

$$
\begin{gathered}
D \tilde{\eta}_{0 x x}-\tilde{\eta}_{0}=0, \quad-1<x<1 ; \quad \tilde{\eta}_{0 x}( \pm 1)=0, \\
{\left[D \tilde{\eta}_{0}\right]_{j}=-\frac{c_{j}}{a_{g}} ; \quad\left[D \tilde{\eta}_{0 x}\right]_{j}=\frac{-s}{a_{g}}\left\langle\tilde{\eta}_{0}\right\rangle_{j}, \quad j=1, \ldots, k .}
\end{gathered}
$$

Here $s$, which parametrizes the equilibrium solution branches, is defined in 2.11.

A problem similar to 3.25) was formally derived in Proposition 8 of [17] in the context of the GM model (1.4) with exponent set $(p, q, m, s)$, where $\zeta \equiv q m /(p-1)-(1+s)>0$. By comparing Proposition 8 of [17] with Principal Result 3.1 above, we conclude that 3.25) for the GS model is equivalent to the corresponding problem for the small eigenvalues of a GM model with exponent set $(p, q, m, s)=(2, s, 2, s)$, where $s=\left(1-U_{ \pm}\right) / U_{ \pm}$. A similar spectral equivalence principle for the large eigenvalues of the GM model and the GS model in the low feed-rate regime was derived in Proposition 3.3 of [21].

To calculate explicit formulae for the small eigenvalues, we must first solve $\sqrt{3.25}$ to calculate $\left\langle\tilde{\eta}_{0 x}\right\rangle_{j}$ for $j=1, \ldots, k$. By substituting the resulting expressions into 3.24 we find that $\lambda_{j}$, for $j=$ $1, \ldots, k$, are the real eigenvalues of a certain generalized symmetric matrix eigenvalue problem. 
Each eigenvector of this problem generates a set of coefficients $c_{j}$ for $j=1, \ldots, k$. The eigenvalues and eigenvectors of this matrix can be calculated analytically. Since the details of this exact, but lengthy, calculation parallel that given in $\$ 4.2$ of [17] for the GM model (1.4], we only outline the key steps in this derivation in Appendix B. In this way, the following rigorous result is obtained for the small eigenvalues of Principal Result 3.1.

Proposition 3.2 Let $\varepsilon \ll 1$ and $\tau=O(1)$. Assume that the invertibility condition (B.12) holds. Then the eigenvalues of 2.13) of order $O\left(\varepsilon^{2}\right)$ are given explicitly by

$$
\lambda_{j} \sim-\frac{2 \varepsilon^{2} s}{D}\left[\frac{1-\cos (\pi j / k)-z_{j}\left(\cosh \left(2 \theta_{0} / k\right)-1\right)}{\cosh \left(2 \theta_{0} / k\right)-\cos (\pi j / k)}\right], \quad j=1, \ldots, k .
$$

Here $z_{j}$ is defined in terms of the parametrization $s=\left(1-U_{ \pm}\right) / U_{ \pm}$of each $k$-spike equilibrium solution branch as

$$
z_{j} \equiv \frac{s \gamma}{s \gamma-\xi_{j}} \operatorname{csch}^{2}\left(\frac{2 \theta_{0}}{k}\right) \sin ^{2}\left(\frac{\pi j}{k}\right), \quad j=1, \ldots, k ; \quad \gamma \equiv 2 \tanh \left(\frac{\theta_{0}}{k}\right), \quad \theta_{0}=D^{-1 / 2} .
$$

For $j=1, \ldots, k, \xi_{j}$ is defined in B.20a) of Appendix B. The corresponding eigenfunction $\phi$ from (3.5) is

$$
\phi(x) \sim \sum_{n=1}^{k}\left(c_{n} w^{\prime}\left[\varepsilon^{-1}\left(x-x_{n}\right)\right]+\varepsilon s\left\langle\tilde{\eta}_{0}\right\rangle_{n} w\left[\varepsilon^{-1}\left(x-x_{n}\right)\right]\right),
$$

where $\left\langle\tilde{\eta}_{0}\right\rangle_{n}$ is the $n^{\text {th }}$ component of $\left\langle\tilde{\boldsymbol{\eta}}_{0}\right\rangle$ given by

$$
\left\langle\tilde{\boldsymbol{\eta}}_{0}\right\rangle=\gamma \sqrt{D}\left[I+s \gamma(\mathcal{B}-s \gamma I)^{-1}\right] \mathcal{P}_{g} c .
$$

Here the matrices $\mathcal{B}$ and $\mathcal{P}_{g}$ are defined in $\overline{B .10}$ and $\bar{B}$.4 , respectively. Finally, there are $k$ independent choices for the vector of coefficients $c^{t}=\left(c_{1}, \ldots, c_{k}\right)$, given explicitly by $\boldsymbol{c}_{j}^{t}=$ $\left(c_{1, j}, \ldots, c_{k, j}\right)$ where

$$
c_{k}^{t}=\frac{1}{\sqrt{k}}\left(1,-1,1, \ldots,(-1)^{k+1}\right) ; \quad c_{l, j}=\sqrt{\frac{2}{k}} \sin \left(\frac{\pi j}{k}(l-1 / 2)\right), \quad j=1, \ldots, k-1 .
$$

Here $c_{j}^{t}$, for $j=1, \ldots, k$, are the eigenvectors of the matrix $\mathcal{B}_{g}$ defined in B.20b.

As shown in Appendix B, the invertibility condition (B.12) holds when the parameters are such that $\lambda=0$ is not an eigenvalue of the NLEP problem A.1 of Appendix A for the large eigenvalues.

Next, we determine the sign of $\lambda_{j}$ with respect to the parameters. Since $z_{k}=0$ in $3.26 \mathrm{~b}$, it follows that $\lambda_{k}<0$ for any $k, D$ and $\mathcal{A}$. Therefore, there is always one negative eigenvalue and one stable direction, given by $c_{k}$ in (3.28) and 3.27a), for translational perturbations of the equilibrium solution. From 3.26a) we infer that $\lambda_{j}<0$ for $j=1, \ldots, k-1$ if and only if

$$
1-\cos \left(\frac{\pi j}{k}\right)-2 z_{j} \sinh ^{2}\left(\frac{\theta_{0}}{k}\right)>0, \quad j=1, \ldots, k .
$$

Using (3.26b) for $z_{j}$, we can write 3.29 ) as

$$
\frac{\xi_{j}}{\gamma}<s-s \cos ^{2}\left(\frac{\pi j}{2 k}\right) \operatorname{sech}^{2}\left(\frac{\theta_{0}}{k}\right), \quad j=1, \ldots, k .
$$


Finally, by B.20a for $\xi_{j}$, a short calculation shows that we can write the stability condition 3.30 as

$$
\left(1-s+\operatorname{csch}^{2}\left(\frac{\theta_{0}}{k}\right)\right)\left(1-\cos ^{2}\left(\frac{\pi j}{2 k}\right) \operatorname{sech}^{2}\left(\frac{\theta_{0}}{k}\right)\right)<0, \quad j=1, \ldots, k .
$$

Since the second bracketed term on the left-hand side of 3.31 is always positive, we conclude that $\lambda_{j}<0$ for $j=1, \ldots, k-1$ if and only if

$$
1-s+\operatorname{csch}^{2}\left(\frac{\theta_{0}}{k}\right)<0 .
$$

Since (3.32) is independent of $j$, it follows that any zero-crossing of the small eigenvalues $\lambda_{j}$, for $j=1, \ldots, k-1$, must be simultaneous.

For the small solution $u_{+}, v_{+}$, where $0<s<1$, the left-hand side of (3.32) is always positive, and so there are $k-1$ positive small eigenvalues $\lambda_{j}$, for $j=1, \ldots, k-1$. For the large solution where $s>1$, we can calculate stability thresholds from 3.32. The threshold condition from 3.32) can be written as

$$
\sinh ^{2}\left(\frac{\theta_{0}}{k}\right)=\frac{1}{s-1}, \quad \theta_{0} \equiv D^{-1 / 2} .
$$

This can be solved in the form

$$
D=\frac{4}{k^{2}}\left(\ln \left[\left(\frac{s+1}{s-1}\right)+\sqrt{\left(\frac{s+1}{s-1}\right)^{2}-1}\right]\right)^{-2}
$$

In (3.34, we then use 2.11 for $s$ to write $D$ in terms of $\mathcal{A}$. This yields the following main result:

Proposition 3.3 Let $\varepsilon \ll 1$, and $\tau=O(1)$. For $k=1$, both the large and small solutions $u_{ \pm}, v_{ \pm}$are always stable with respect to the small eigenvalue. For $k>1$, and for the small solution $u_{+}, v_{+}$, there are always $k-1$ positive small eigenvalues and one negative eigenvalue $\lambda_{k}$. Similarly, for $k>1$, we also have $\lambda_{k}<0$ for the large solution $u_{-}, \nu_{-}$. However, the other small eigenvalues $\lambda_{j}$, for $j=1, \ldots, k-1$, are negative at a fixed value of $\mathcal{A} / \mathcal{A}_{k e}$ if and only if $D$ satisfies

$$
D<D_{k S} \equiv \frac{4}{k^{2}\left[\ln \left(r+\sqrt{r^{2}-1}\right)\right]^{2}}, \quad r \equiv\left[1-\frac{\mathcal{A}_{k e}^{2}}{\mathcal{A}^{2}}\right]^{-1 / 2} .
$$

If $D=D_{k S}$, then $\lambda=0$ is an eigenvalue of algebraic multiplicity $k-1$.

The criterion 3.35 expresses $D_{k s}$ in terms of the ratio $\mathcal{A}_{k e} / \mathcal{A}$. However, since $\mathcal{A}_{k e}$ also depends on $D$, the criterion 3.35 is a transcendental equation for $D_{k S}$. Below, we solve this equation asymptotically in the intermediate regime to obtain a certain scaling law.

A similar criterion can be found with respect to $\mathcal{A}$. We substitute the stability condition 3.32 into 2.11 to obtain the stability threshold

$$
\frac{\mathcal{A}}{\mathcal{A}_{k e}}=\frac{1+s}{2 \sqrt{s}}=\frac{2+\operatorname{csch}^{2}\left(\theta_{0} / k\right)}{2 \sqrt{1+\operatorname{csch}^{2}\left(\theta_{0} / k\right)}} .
$$

By simplifying (3.36), we obtain the next result. 
PROPOSITION 3.4 Let $\varepsilon \ll 1, \tau=O(1)$, and $k>1$. For the large solution $u_{-}, \nu_{-}$, the small eigenvalues $\lambda_{j}$, for $j=1, \ldots, k-1$, are negative at a fixed value of $D$ if and only if $\mathcal{A}$ satisfies

$$
\mathcal{A}>\mathcal{A}_{k S}, \quad \mathcal{A}_{k S} \equiv \mathcal{A}_{k e}\left[\tanh \left(\frac{2 \theta_{0}}{k}\right)\right]^{-1}, \quad \theta_{0} \equiv D^{-1 / 2}
$$

Here $\mathcal{A}_{k e}$ is the existence threshold of 2.9). Notice that $\mathcal{A}_{k S}-\mathcal{A}_{k e} \rightarrow 0^{+}$as $D \rightarrow 0$.

Propositions 3.3 and 3.4 show that $k-1$ small eigenvalues simultaneously cross through zero as either $D$ crosses through $D_{k S}$ or as $\mathcal{A}$ crosses through $\mathcal{A}_{k S}$. Therefore, there must be a bifurcation at these critical values. In $\S 4$, this bifurcation is shown to be related to the emergence of asymmetric solution branches.

Next, we compare $D_{k S}$ and $\mathcal{A}_{k S}$ with the large eigenvalue stability thresholds $D_{k L}$ and $\mathcal{A}_{k L}$ of Principal Result A.2, which hold when $\tau$ is below the Hopf bifurcation threshold. A simple calculation shows that

$$
\mathcal{A}_{k S}>\mathcal{A}_{k L}, \quad D_{k S}<D_{k L} .
$$

Therefore, the stability thresholds with respect to the small eigenvalues are more stringent than those for the large eigenvalues. The next result summarizes the main stability conclusion for the large solution branch $u_{-}$and $v_{-}$with respect to both the small and the large eigenvalues.

PRINCIPAL RESUlt 3.5 Let $\varepsilon \ll 1$, and consider the large solution branch $u_{-}$, $v_{-}$of Principal Result 2.1. Suppose that $\tau<\tau_{h L}$, where $\tau_{h L}$ is the Hopf bifurcation value of the NLEP problem of Principal Result A.1 for the large eigenvalues. Then the $k$-spike symmetric solution branch is stable with respect to both the large and the small eigenvalues when either $D<D_{k S}$, or equivalently when $\mathcal{A}>\mathcal{A}_{k S}$. For $\tau<\tau_{h L}$ where $D$ satisfies $D_{k S}<D<D_{k L}$, or when $\mathcal{A}_{k L}<\mathcal{A}<\mathcal{A}_{k S}$, the large solution is stable with respect to the large eigenvalues but is not stable with respect to the small eigenvalues.

To illustrate our main stability result graphically, in Fig. 1 (b) of $\S 1$ we plotted the norm $|v|_{2}$ defined in 2.10 versus $\mathcal{A}$ for the parameter values $D=0.75$ and $k=1, \ldots, 4$. The stability information of Principal Result 3.5 is shown by different shadings of different portions of these branches. For smaller values of $D$, we still have $\mathcal{A}_{k e}<\mathcal{A}_{k L}<\mathcal{A}_{k S}$ for $k \geqslant 2$, but in this case $\mathcal{A}_{k S}$ is close to $\mathcal{A}_{k e}$.

Next, we derive a scaling law for the small eigenvalues that is valid in the intermediate parameter regime $O(1) \ll \mathcal{A} \ll O\left(\varepsilon^{-1 / 2}\right)$. In this regime, we know from 2.11 that to leading order, $s \sim$ $4 \mathcal{A}^{2} / \mathcal{A}_{k e}^{2} \gg 1$. Letting $s \rightarrow \infty$ in 3.26 we obtain the following limiting result for the small eigenvalues:

Proposition 3.6 Let $\varepsilon \ll 1, D=O(1), \tau=O(1)$, and assume that $O(1) \ll \mathcal{A} \ll O\left(\varepsilon^{-1 / 2}\right)$. Then, for the large solution $u_{-}, \nu_{-}$, the small eigenvalues of 2.13 are given asymptotically by

$\lambda \sim-\frac{4 \varepsilon^{2} \mathcal{A}^{2} \theta_{0}}{3} \tanh \left(\frac{\theta_{0}}{k}\right) \sin ^{2}\left(\frac{\pi j}{2 k}\right) \frac{1-\operatorname{sech}^{2}\left(\theta_{0} / k\right) \cos ^{2}(\pi j /(2 k))}{\cosh \left(2 \theta_{0} / k\right)-\cos (\pi j / k)}+O\left(\varepsilon^{2}\right), \quad j=1, \ldots, k$.

From (3.39), we conclude that $\lambda_{j}<0$ for $j=1, \ldots, k$ when $D=O(1)$ and $\tau=O(1)$. Therefore, when $D=O(1)$ and $\tau=O(1)$, we always have stability with respect to the small eigenvalues in the intermediate regime for $\mathcal{A}$. However, stability can be lost when $D$ is asymptotically large. To 
show this we use $s \sim 4 \mathcal{A}^{2} / \mathcal{A}_{k e}^{2}$ in 3.35 to conclude that a $k$-spike pattern is stable with respect to translations when $D<D_{k S}^{\infty}$, where $D_{k S}^{\infty}$ for $k>1$ is the unique root of

$$
\sqrt{D}=\frac{\mathcal{A}^{2}}{3 k^{2}} \tanh \left(\frac{1}{k \sqrt{D}}\right)
$$

In the intermediate regime, 3.40 can be solved asymptotically to predict a minimum inter-spike distance $L_{m S}$ for the translational stability of a spike pattern. For a $k$-spike pattern on a domain of length 2, the inter-spike separation is $L=2 / k$. For $\mathcal{A} \gg 1$, the root of 3.40 ) satisfies $D \gg 1$. For $D \gg 1$, we solve 3.40 for $k$ with $\mathcal{A}=\varepsilon^{-1 / 2} A$ to conclude that a $k$-spike pattern is translationally stable when

$$
L>L_{m S} \sim\left(\frac{24 D \varepsilon}{A^{2}}\right)^{1 / 3}, \quad O\left(\varepsilon^{1 / 2}\right) \ll A \ll O(1) .
$$

Since $L_{m S} \gg O(\varepsilon)$, the calculation is self-consistent. A similar scaling law for competition instabilities in the intermediate regime, associated with the large eigenvalues, was derived in $\S 4$ of [21]. For $\tau$ below the Hopf bifurcation threshold $\tau_{h L}$, it was shown in [21] that there are no positive real eigenvalues when

$$
L>L_{m C} \sim\left(\frac{12 \gamma_{k} D \varepsilon}{A^{2}}\right)^{1 / 3}, \quad O\left(\varepsilon^{1 / 2}\right) \ll A \ll O(1) ; \quad \gamma_{k}=1+\cos (\pi / k) .
$$

Clearly $L_{m C}<L_{m S}$. Therefore, as the inter-spike separation distance $L$ is decreased below $L_{m S}$, instability is first lost due to $k-1$ independent slow translational instabilities. As $L$ is further decreased below $L_{m C}$, there are fast $O(1)$ instabilities leading to spike annihilation events.

Finally, we comment on the uniformity of our results in (3.26) and (3.39) with respect to the diffusivity $D$, which measures the inter-spike interaction strength. First, we consider a one-spike solution with $D \gg 1$. From 3.26 , we obtain $\lambda_{1} \sim-2 \varepsilon^{2} s / D<0$ for $D \gg 1$. This suggests that a one-spike solution to the GS model is stable when $D \gg 1$. However, for the shadow problem where $D=\infty$, it is well known for the corresponding GM model (1.4) that a spike is translationally unstable for $\varepsilon \ll 1$ due to a positive exponentially small eigenvalue, which leads to dynamic metastability of the spike (cf. [17], [20]). Therefore, with the close relationship between the GS and GM models, 3.26 for the GS model is not expected to be uniformly valid as $D \rightarrow \infty$. In fact, for $D=O\left(e^{c / \varepsilon}\right)$ an extra term must be added to the expression $\lambda_{1} \sim-2 \varepsilon^{2} s / D$, which arises from analyzing exponentially weak boundary layer effects for $\phi$ near $x= \pm 1$. The resulting formula for $\lambda_{1}$ leads to a bifurcation of a one-spike equilibrium on the range $D=O\left(e^{c / \varepsilon}\right)$, for some $c>0$. We do not study this problem in detail here, since a very similar issue for the GM model (1.4) was studied in detail in [20] (see also $\$ 5.2$ of [17]).

For the GS model in the other limit where $D \rightarrow 0$, we summarize our comments as follows:

REMARK 3.1 The analysis leading to 3.26 and 3.39 is valid provided that $D \gg O\left(\varepsilon^{2}\right)$. This is needed to ensure a decoupling of the slow and fast variables in the core of the spike. However, if we did let $D \rightarrow 0$ in $\left(3.26\right.$, but with $D \gg O\left(\varepsilon^{2}\right)$, after a short calculation we obtain

$\lambda_{k} \sim-\frac{8 \varepsilon^{2} s}{D} e^{-2 /(k \sqrt{D})} ; \quad \lambda_{j} \sim \frac{8 \varepsilon^{2} s}{D}\left(\frac{s}{s-1}\right) \sin ^{2}\left(\frac{\pi j}{k}\right) e^{-2 /(k \sqrt{D})}, \quad j=1, \ldots, k-1$.

Therefore, for $D \ll 1$, there are $k$ exponentially small eigenvalues, with $k-1$ of them being positive for the large solution branch where $s>1$. However, in the weak interaction regime $D=$ 
$O\left(\varepsilon^{2}\right)$, where both variables are localized, a $k$-spike pattern with an $O(1)$ inter-spike separation distance is essentially equivalent to a periodic, near-homoclinic, pattern of an asymptotically large period. For such long-wavelength periodic patterns, with a sufficiently large wavelength, it was proved in [12] that there is a continuous loop of spectra in the neighborhood of each isolated eigenvalue of the infinite-period homoclinic connection. Although the result (3.43) was derived for Neumann boundary conditions rather than periodic conditions, the discrete spectra in (3.43) should presumably be related to the breaking of the spectral loop near the origin resulting from the transition from the weak to the semi-strong interaction regime. A potential parametrization for this transition is to take $D=\varepsilon^{2-p}$ with $0 \leqslant p \leqslant 2$.

\subsection{Slow translational instabilities: dynamics}

We now perform a few numerical experiments to illustrate the theory and to show the significance of the small eigenvalues for the dynamics of spike patterns. The computational results shown below for (1.3) have been computed using the routine D03PCF of the NAG library [31] with 1500 uniformly spaced meshpoints.

For (1.3) we take $D=0.75, k=2, \varepsilon=0.03$, and $\tau=2.0$. For this two-spike equilibrium solution, the stability thresholds for the small and large eigenvalues, obtained from 3.37) and A.4, respectively, are $\mathcal{A}_{2 S}=6.296$ and $\mathcal{A}_{2 L}=5.633$. Since $\tau$ is below the stability threshold $\tau_{h L}$, there is no Hopf bifurcation for the equilibrium profile. For the initial condition for (1.3), we take the equilibrium solution of Principal Result 2.1, with initial spike locations $x_{j}(0)$ slightly offset from their equilibrium values of $\pm 1 / 2$.

We first take $\mathcal{A}=6.5>\mathcal{A}_{2 S}$, and $x_{1}(0)=0.52, x_{2}(0)=-0.48$. For this value of $\mathcal{A}$, the two small eigenvalues are negative. Therefore, the equilibrium solution should be stable with respect to translations of the profile. In Fig. 3(a) we plot the spike amplitudes $v_{m}(t)=v_{-}\left(x_{j}, t\right)$, for $j=1,2$, versus $t$ showing the convergence towards a symmetric two-spike equilibrium solution over a long time-scale. In Fig. 3(b) we plot $x_{2}$ versus $t$, which shows that $x_{2} \rightarrow 1 / 2$ as $t$ increases. A similar plot can be made for $x_{1}$.

Next, we take $\mathcal{A}=6.0$, so that $\mathcal{A}_{2 L}<\mathcal{A}<\mathcal{A}_{2 S}$. We again choose $x_{1}(0)=0.52, x_{2}(0)=$ -0.48 . In Fig. 4(a) we plot the locations of the two spikes as a function of time showing the

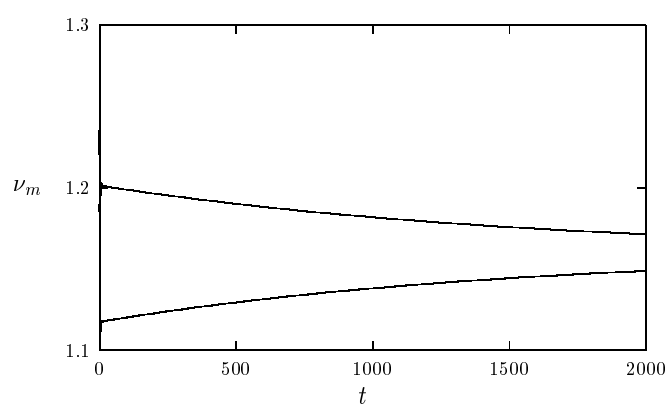

(a) $v_{m}$ versus $t$

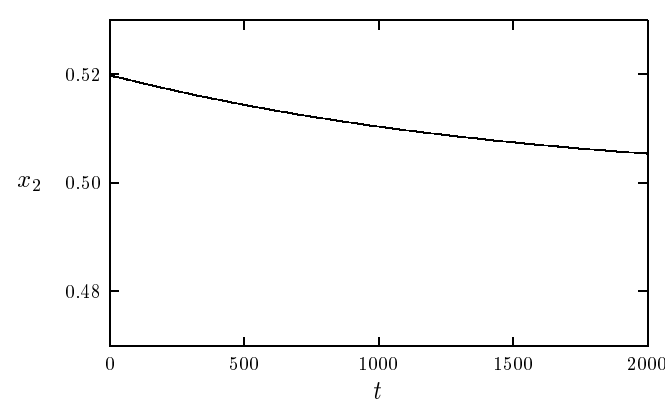

(b) $x_{j}$ versus $t$

FIG. 3. Numerical solution to 1.3 with $D=0.75, k=2, \varepsilon=0.03, \tau=2.0$, and $\mathcal{A}=6$.5. Left figure: plot of the spike amplitudes $v_{m}$ of $v_{-}$versus $t$. Right figure: plot of the spike location $x_{2}$ versus $t$. 


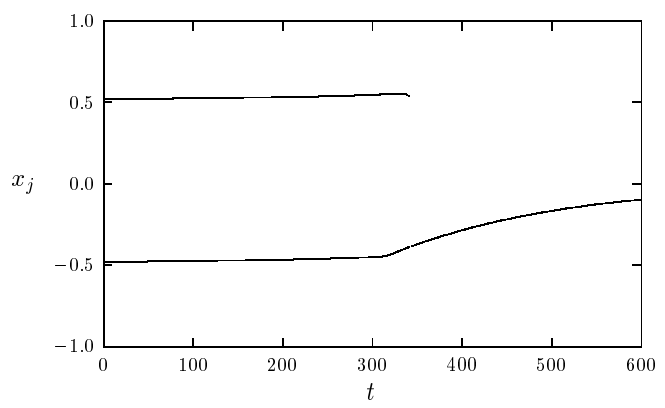

(a) $x_{j}$ versus $t$

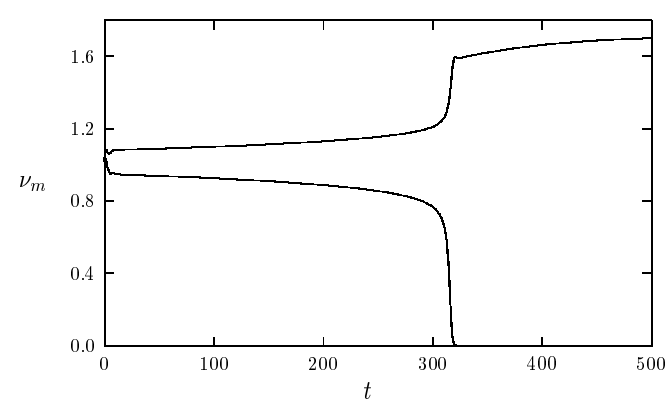

(b) $v_{m}$ versus $t$

FIG. 4. Numerical solution to 1.3 with $D=0.75, k=2, \varepsilon=0.03, \tau=2.0$, and $\mathcal{A}=6.0$. Left figure: plot of the spike locations $x_{j}$ versus $t$. Right figure: plot of the spike amplitudes $v_{m}$ versus $t$.

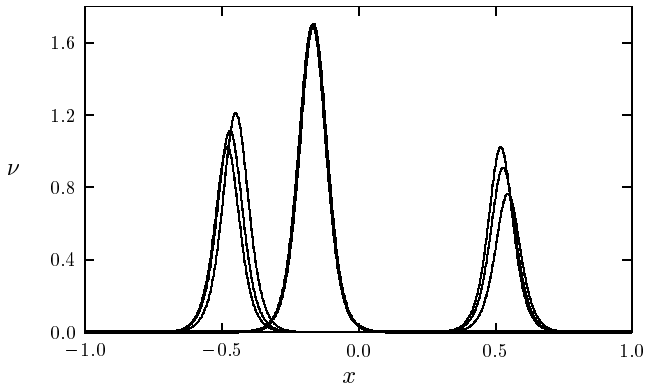

(a) $v$ versus $x$

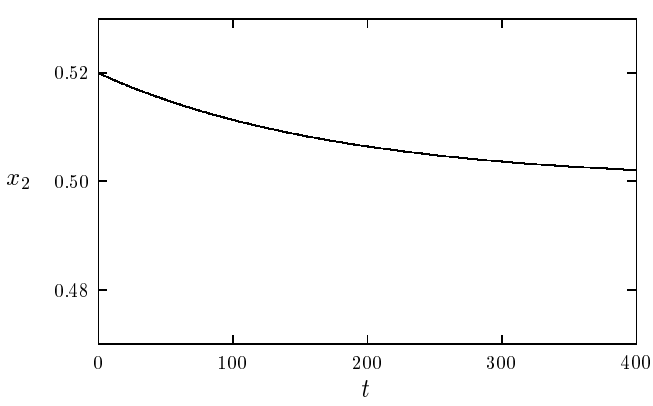

(b) $x_{2}$ versus $t$

FIG. 5. Left figure: plot of numerical solution to 1.3 at different times for the parameter values of Fig. 4 The clustered solid curves correspond to $t=0,150,300$, and the heavy solid curve corresponds to $t=500$. Only one spike remains at $t=500$. Right figure: plot of the spike location $x_{2}$ versus $t$ for the parameter values of Fig. 4 except now with symmetric initial locations $x_{1}(0)=0.52$ and $x_{2}(0)=-0.52$.

divergence away from the two-spike equilibrium locations $\pm 1 / 2$. In Fig. 4(b) we plot the amplitudes $v_{m}$ of the two spikes versus $t$. The numerical solution for $v$ versus $x$ is shown in Fig. 5(a) at different times. This example suggests the following scenario: For this value of $\mathcal{A}=6.0$, the equilibrium twospike solution is stable with respect to profile instabilities (large eigenvalues), but is unstable with respect to translations (small eigenvalues). Therefore, the locations of the two spikes diverge away from $\pm 1 / 2$. During their evolution, a competition instability occurs on a fast $O(1)$ time-scale as a result of a large eigenvalue crossing into the right half-plane. Recall that the results in $\S 2$ and Appendix A pertain only to profile instabilities associated with equilibrium spike solutions, and not the quasi-equilibrium solutions where the spike locations are not at their equilibrium positions. This competition instability annihilates one of the spikes at approximately $t \approx 500$, and the other spike then slowly drifts to the stable one-spike equilibrium solution centered at the origin. Since $\lambda_{k}<0$ in Proposition 3.3, a one-spike equilibrium solution is always stable with respect to translations.

Finally, we take $\mathcal{A}=6.0$, but we now introduce a symmetric perturbation in the initial spike locations so that $x_{1}(0)=-0.52$ and $x_{2}(0)=0.52$. Although, the equilibrium solution is unstable 
with respect to translations, the spike location $x_{2}$ is shown in Fig. $5(\mathrm{~b})$ to approach its equilibrium value. An identical convergence occurs for $x_{1}$. To explain this, we recall from Proposition 3.3 that, although $\lambda_{1}>0$ for $\mathcal{A}=6.0$, we always have $\lambda_{2}<0$. Therefore, there is a saddle-structure for the two-spike equilibrium solution. From Proposition 3.3, and from the form of the stable eigenvector $\boldsymbol{c}_{2}$, given in (3.28) with $k=2$, it follows that symmetric perturbations of the initial spike locations are, locally, on the stable manifold of the saddle point. Therefore, with sufficient numerical resolution it may be possible that we can approach the saddle point as $t$ increases. This is what is observed in Fig. 5(b)

\section{Asymmetric $k$-spike equilibria}

We now construct asymmetric equilibrium spike patterns, where the spikes have different heights. We follow a similar approach as in [45] for the GM model (1.4) by first constructing a one-spike solution centered at the origin for (1.3) posed on $-l<x<l$, with $u_{x}( \pm l)=v_{x}( \pm l)=0$. For $\varepsilon \ll 1$, such a solution for $v$ and $u$ is even, $v(l)$ is exponentially small, and $u(l)=O(1)$. We determine all values of $l$, labeled by $l_{1}, \ldots, l_{n}$, such that $u\left(l_{1}\right)=\cdots=u\left(l_{n}\right)$. For a certain range of the parameters, as found below, there are exactly two such values of $l$. These "local" solutions are then used to construct a global asymmetric equilibrium $k$-spike pattern for $(1.3)$ on $[-1,1]$.

To construct a solution to (1.3) on $-l<x<l$ with a spike at the origin, we proceed as in $\$ 2$ for a symmetric spike pattern. We obtain

$$
v(x) \sim \frac{1}{\mathcal{A} U} w(x / \varepsilon), \quad u(x) \sim 1-\frac{6}{\mathcal{A}^{2} U} G_{l}(x ; 0) .
$$

Here $w(y)$ satisfies $[2.2]$, and $G_{l}(x ; 0)$ is the Green's function on $-l<x<l$ satisfying

$$
D G_{l x x}-G_{l}=-\delta(x), \quad-l<x<l ; \quad G_{l x}( \pm l ; 0)=0 ; \quad G_{l}(x ; 0)=\left(\frac{\theta_{0}}{2}\right) \frac{\cosh \left[(l-|x|) \theta_{0}\right]}{\sinh \left(l \theta_{0}\right)},
$$

where $\theta_{0} \equiv D^{-1 / 2}$. The constant $U$ in 4.1 , representing the leading-order approximation for $u$ in the inner region, is obtained by setting $u(0)=U$ in 4 .1. By solving the resulting quadratic equation we get

$$
U=\frac{1}{2}\left(1 \pm \sqrt{1-\frac{\operatorname{coth}\left(\theta_{0} l\right)}{\tilde{\mathcal{A}}^{2}}}\right), \quad \mathcal{A} \equiv \sqrt{12 \theta_{0}} \tilde{\mathcal{A}}, \quad \theta_{0} \equiv D^{-1 / 2} .
$$

To construct asymmetric patterns, we must calculate $u(l)$. Using (4.1) and (4.2), we get

$$
\frac{u(l)-1}{U-1}=\frac{G_{l}(l ; 0)}{G_{l}(0 ; 0)}=\operatorname{sech}\left(l \theta_{0}\right) .
$$

Combining (4.4) and (4.3), we obtain a key formula for $u(l)$ :

$$
u(l)=E_{ \pm}(z) \equiv 1+\frac{1}{2} \operatorname{sech}(z)\left[-1 \pm \sqrt{1-\frac{\operatorname{coth} z}{\tilde{\mathcal{A}}^{2}}}\right], \quad z \equiv \theta_{0} l .
$$


The minus and plus signs in 4.5 refer to the large and small solutions, respectively. In 4.5$), E_{ \pm}(z)$ is defined only when $\tilde{\mathcal{A}}^{2} \geqslant \operatorname{coth} z>1$. This yields $z>z_{0}$, where

$$
z_{0} \equiv \frac{1}{2} \ln \left(\frac{\tilde{\mathcal{A}}^{2}+1}{\tilde{\mathcal{A}}^{2}-1}\right)
$$

Clearly $E_{ \pm}(z)>0$ for $z>z_{0}$. For $z>z_{0}$, we readily derive some key properties of $E_{ \pm}(z)$.

LEMmA 4.1 Let $\tilde{\mathcal{A}}>1$. Then, for $z>z_{0}, E_{+}^{\prime}(z)>0$ with $E_{+}\left(z_{0}\right)<1$, and $E_{+}(z) \rightarrow 1$ as $z \rightarrow \infty$. Alternatively, $E_{-}\left(z_{0}\right)<1$ with $E_{-}^{\prime}(z)<0$ for $z_{0}<z<z_{m}$, and $E_{-}^{\prime}(z)>0$ for $z>z_{m}$. Moreover, $E_{-}(z) \rightarrow 1$ as $z \rightarrow \infty$. The point $z_{m}$ where $E_{-}(z)$ has its minimum value is the unique root of

$$
\tilde{\mathcal{A}}=[\tanh z]^{-1 / 2}[\tanh (2 z)]^{-1} \text {. }
$$

Proof. Clearly $E_{ \pm}\left(z_{0}\right)<1$ and $E_{ \pm}(z) \rightarrow 1$ as $z \rightarrow \infty$. In addition, by differentiating (4.5) it follows readily that $E_{+}^{\prime}(z)>0$ for $z>z_{0}$. For $E_{-}(z)$, we calculate

$$
E_{-}^{\prime}(z)=\frac{\sinh z}{2 \cosh ^{2} z}\left[1+\sqrt{1-\frac{\operatorname{coth} z}{\tilde{\mathcal{A}}^{2}}}\right]-\frac{\operatorname{sech} z}{\tilde{\mathcal{A}}^{2}} \frac{\operatorname{csch}^{2} z}{\sqrt{1-\frac{\operatorname{coth} z}{\tilde{\mathcal{A}}^{2}}}} .
$$

Therefore, $E_{-}^{\prime}(z)<0$ only when

$$
2 \tilde{\mathcal{A}}^{2} \tanh z\left[\sqrt{1-\frac{\operatorname{coth} z}{\tilde{\mathcal{A}}^{2}}}+1-\frac{\operatorname{coth} z}{\tilde{\mathcal{A}}^{2}}\right]>\operatorname{csch}^{2} z .
$$

By manipulating 4.9 , we find that $E_{-}^{\prime}(z)<0$ if and only if

$$
\sqrt{1-\frac{\operatorname{coth} z}{\tilde{\mathcal{A}}^{2}}}>\frac{\cosh (2 z)}{2 \tilde{\mathcal{A}}^{2} \sinh ^{2} z} \operatorname{coth} z-1
$$

It is easy to see from 4.10 that there exists a $z_{m}$ such that $E_{-}^{\prime}(z)<0$ for $z_{0}<z<z_{m}$, and $E_{-}^{\prime}(z)>0$ for $z>z_{m}$. To determine where $E_{-}^{\prime}(z)=0$, we square both sides of 4.10 to obtain

$$
1-t=\left(\frac{t \cosh (2 z)}{2 \sinh ^{2} z}-1\right)^{2}, \quad t \equiv \frac{\operatorname{coth} z}{\tilde{\mathcal{A}}^{2}} .
$$

By solving 4.11) for $t>0$ we find that $t=\tanh ^{2}(2 z)$. Since $\tilde{\mathcal{A}}^{2}=(\operatorname{coth} z) / t$, we obtain 4.7 ) for $\tilde{\mathcal{A}}$.

Therefore, when $\tilde{\mathcal{A}}>1$, it follows that for any $z$ in $z_{0}<z<z_{m}$, there exists a unique $\tilde{z}$, with $\tilde{z}>z_{m}$, such that $E_{-}(z)=E_{-}(\tilde{z})$. In Fig. 6 we plot $E_{-}(z)$ versus $z$ when $\tilde{\mathcal{A}}=3$. Since $z=\theta_{0} l$ and $\tilde{z}=\theta_{0} \tilde{l}$, the implication of this result is that given any $l$ with $z_{0}<l \theta_{0}<z_{m}$, there exists a unique $\tilde{l}$, with $\tilde{l} \theta_{0}>z_{m}$, such that $u(l)=u(\tilde{l})$. This implies that in any asymmetric pattern generated using $E_{-}(z)$ there are only two distinct types of spikes. Furthermore, since $E_{+}(z)$ is increasing we cannot construct asymmetric patterns for the small solution.

For $E_{-}(z)$, solutions of length $l$ and $\tilde{l}$ are called $S$-type and $B$-type spikes, respectively. We now construct asymmetric $k$-spike patterns to the global problem $[1.3]$ on $[-1,1]$ with $k_{1}>0 S$-type 


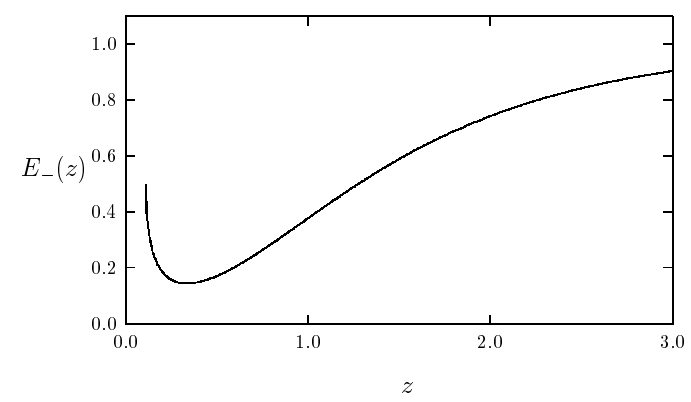

FIG. 6. Plot of $E_{-}$versus $z$ for $z \geqslant z_{0}$ when $\tilde{\mathcal{A}}=3$.

spikes and $k_{2}=k-k_{1}>0 B$-type spikes arranged in any sequence from left to right across the interval as

$$
\operatorname{SBSSB} \ldots B, \quad k_{1} S \text { 's, } \quad k_{2} B \text { 's. }
$$

To do so, we use translation invariance and the condition $u(l)=u(\tilde{l})$ to glue $S$-type and $B$-type spikes together to obtain differentiability for the global function $u$ on $[-1,1]$. The global function $v$ is asymptotically differentiable since the local function is such that $v(l)$ and $v(\tilde{l})$ are exponentially small when $\varepsilon \ll 1$.

Since the supports of an $S$-spike and a $B$-spike are $2 l$ and $2 \tilde{l}$, respectively, we get the length constraint $2 k_{1} l+2 k_{2} \tilde{l}=2$. The other condition, which ensures that $u$ is differentiable, is that $E_{-}(z)=E_{-}(\tilde{z})$. Using $l=z \theta_{0}$ and $\tilde{l}=\tilde{z} \theta_{0}$, we obtain a nonlinear coupled algebraic system for $z$ and $\tilde{z}$ :

$$
k_{1} z+k_{2} \tilde{z}=\theta_{0}, \quad E_{-}(z)=E_{-}(\tilde{z}) ; \quad l=z \theta_{0}^{-1}, \quad \tilde{l}=\tilde{z} \theta_{0}^{-1}, \quad \theta_{0}=D^{-1 / 2} .
$$

Here $E_{-}(z)$ is defined in 4.5 . In terms of the half-lengths $l$ and $\tilde{l}$ of the supports of the spikes as given in 4.13, we formally obtain the following result for asymmetric $k$-spike patterns:

PRINCIPAL Result 4.2 Let $\tilde{\mathcal{A}}>1$ and $D>0$. Then, for $\varepsilon \rightarrow 0$, an asymmetric equilibrium $k$-spike pattern for (1.3) with $k_{1}$ spikes of type $\mathrm{S}$ and $k_{2}=k-k_{1}$ spikes of type $B$ is characterized by

$$
\nu_{-}(x) \sim \sum_{j=1}^{k} \frac{1}{\mathcal{A} U\left(l_{j}\right)} w\left[\varepsilon^{-1}\left(x-x_{j}\right)\right], \quad U\left(l_{j}\right) \equiv \frac{1}{2}\left(1-\sqrt{1-\frac{\operatorname{coth}\left(\theta_{0} l_{j}\right)}{\tilde{\mathcal{A}}^{2}}}\right), \quad \mathcal{A} \equiv \sqrt{12 \theta_{0}} \tilde{\mathcal{A}}
$$

where $w(y)$ satisfies 2.2). Here for each $j, l_{j}=l$ or $l_{j}=\tilde{l}$, where $l$ and $\tilde{l}$ are determined in terms of $k_{1}, k_{2}, \theta_{0}$, and $\mathcal{A}$ by 4.13 . The value $l_{j}=l$ occurs $k_{1}>0$ times, while $l_{j}=\tilde{l}$ occurs $k_{2}=k-k_{1}>0$ times. The small and large spikes are arranged in any sequence. For $\varepsilon \ll 1$, the leading-order outer solution is

$$
u(x)=1-\sum_{j=1}^{k} \frac{2\left[1-U\left(l_{j}\right)\right]}{\theta_{0} \operatorname{coth}\left(\theta_{0} l_{j}\right)} G\left(x ; x_{j}\right)
$$


Here $G\left(x ; x_{j}\right)$ is the (global) Green's function satisfying 2.5 . The spike locations $x_{j}$ are found from

$$
x_{1}=l_{1}-1, \quad x_{k}=1-l_{k}, \quad x_{j+1}=x_{j}+l_{j+1}+l_{j}, \quad j=1, \ldots, k-2 .
$$

To recover the symmetric $k$-spike equilibrium solutions constructed earlier in $\S 2$, we set $z=\tilde{z}=$ $\theta_{0} / k$, which solves (4.13). Then (4.13) has a solution only when $z=\tilde{z}=z_{m}$. Therefore, setting $z=\theta_{0} / k$ in 4.7 we obtain the critical value of $\tilde{\mathcal{A}}$ for the emergence of the asymmetric branch. By comparing this critical value with that in Proposition 3.4 of $\S 3$, we obtain the following bifurcation result:

PRINCIPAL RESUlt 4.3 Let $k>1$ and $\varepsilon \ll 1$. For a fixed $D>0$ the asymmetric $k$-spike equilibrium solutions bifurcate from the $k$-spike symmetric large equilibrium solution branch of Principal Result 2.1 at the value $\mathcal{A}=\mathcal{A}_{k S}$ in 3.37) of Proposition 3.4. Alternatively, for a fixed ratio $\mathcal{A} / \mathcal{A}_{k e}>1$, the asymmetric $k$-spike branches bifurcate from the large symmetric $k$-spike solution branch at the value $D=D_{k S}$ in 3.35 of Proposition 3.3. These critical values are precisely the thresholds where $k-1$ small eigenvalues associated with the translational stability of the large symmetric solution simultaneously cross through zero.

Neglecting the positioning of the small and large spikes in a $k$-spike sequence, there are $k-1$ possible asymmetric patterns for a $k$-spike solution. Principal Result 4.3 shows that these patterns emerge at the simultaneous zero-crossings of $k-1$ small eigenvalues for the symmetric $k$-spike solution branch.

This result was illustrated graphically in Fig. 1(a) of $\S 1$. To display the bifurcation diagram for the asymmetric branches we use 4.14a to calculate the $L_{2}$ norm $|v|_{2}$ as

$$
|v|_{2} \equiv\left(\varepsilon^{-1} \int_{-1}^{1} v^{2} \mathrm{~d} x\right)^{1 / 2} \sim \frac{1}{\mathcal{A}}\left[\left(6 k_{1} /[U(l)]^{2}\right)+\left(6 k_{2} /[U(\tilde{l})]^{2}\right)\right]^{1 / 2} .
$$

In Fig. 1(a) we plotted $|\nu|_{2}$ versus $\mathcal{A}$ for the symmetric and asymmetric solution branches for $k=$ $1, \ldots, 4$ when $D=0.75$. The dashed lines in Fig. 1(a) of $\S 1$ correspond to plots of (4.15) versus $\mathcal{A}$ for all of the asymmetric branches that emerge from the symmetric branches at the bifurcation values $\mathcal{A}=\mathcal{A}_{k S}$, for $k=1, \ldots, 4$. In this bifurcation diagram, the ordering of spikes on the interval is invisible to the norm (4.15). From (4.13), 4.15), and Fig. 1(a), it can be seen that an asymmetric branch with $k_{1}$ small spikes asymptotes to the symmetric branch with $k-k_{1}$ spikes as $\mathcal{A} \rightarrow \infty$.

In Fig. 7 we plot a few of the asymmetric patterns for $v$ when $D=0.75, \varepsilon=0.02$, and $k=1, \ldots, 4$. These parameters correspond to those in the bifurcation diagram of Fig. 1(a). The plots in Fig. 7 are obtained by first solving (4.13) using Newton's method with different numbers $k_{1}$ of small spikes on the interval and for different values of $\mathcal{A}$. The different patterns for $v$ are then obtained from 4.14).

Finally, we comment on the stability of the asymmetric solutions. Although we do not analyze this problem here, we expect that these branches are all unstable with respect to the small eigenvalues. From numerical computations of a certain matrix eigenvalue problem, it was shown in [45] that the asymmetric spike patterns for the GM model (1.4) are unstable with respect to translations. However, since the asymmetric patterns emerge at the point $\mathcal{A}=\mathcal{A}_{k S}>\mathcal{A}_{k L}$, where the NLEP problem A.1 has no unstable eigenvalues for $\tau<\tau_{h L}$, we would expect by continuity that the asymmetric branches are stable with respect to the large eigenvalues for $\mathcal{A}-\mathcal{A}_{k S}$ sufficiently small and when $\tau$ is below some threshold. 


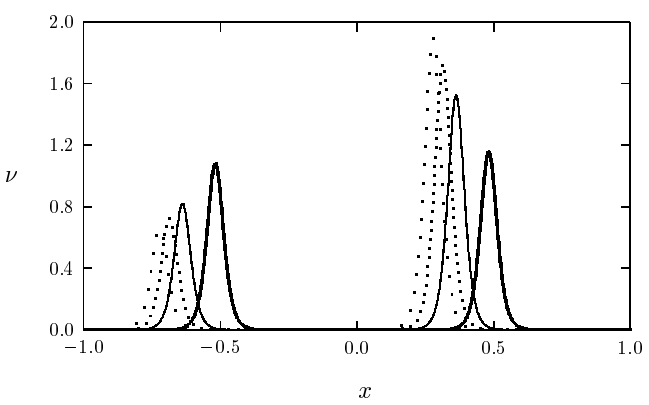

(a) $k=2: S B$ pattern

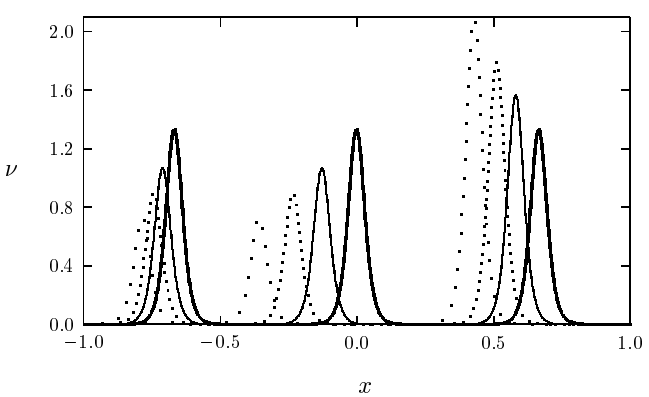

(c) $k=3: S S B$ pattern

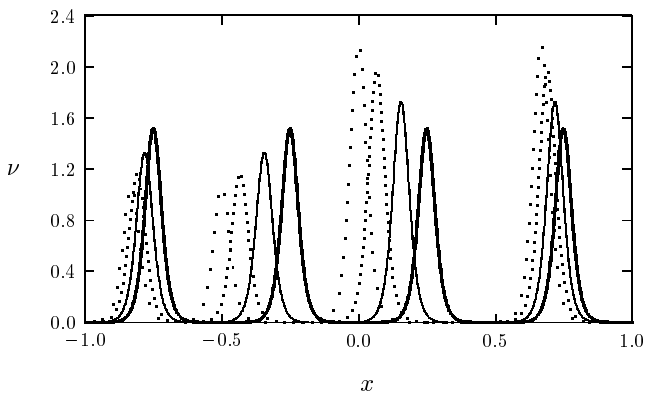

(e) $k=4: S S B B$ pattern

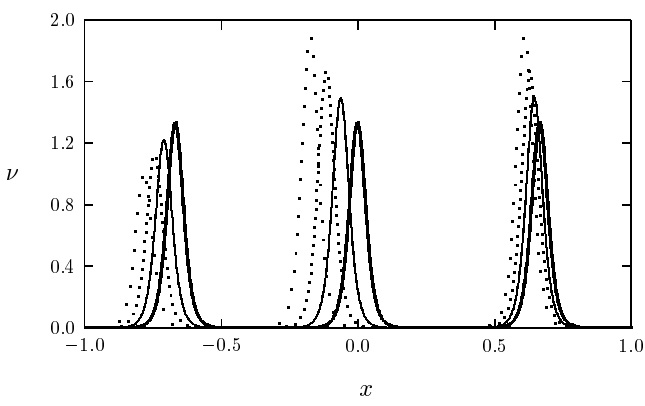

(b) $k=3: S B B$ pattern

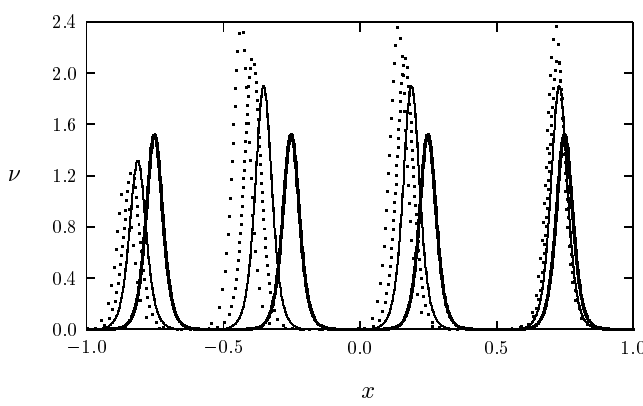

(d) $k=4: S B B B$ pattern

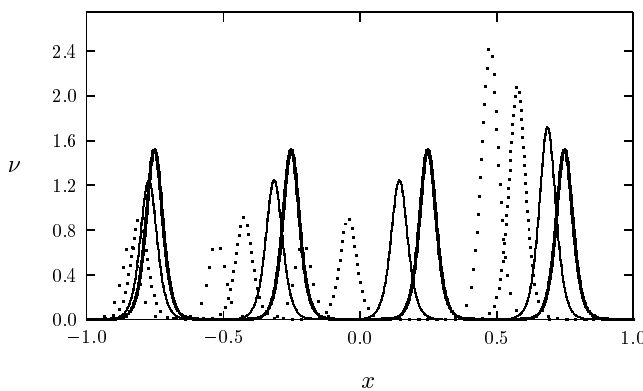

(f) $k=4: \operatorname{SSSB}$ pattern

FIG. 7. Asymmetric patterns for $D=0.75$ and $\varepsilon=0.02$. The thresholds are $\mathcal{A}_{2 S}=6.296, \mathcal{A}_{3 S}=9.500$, and $\mathcal{A}_{4 S}=$ 13.487. Top left: $S B: \mathcal{A}=\mathcal{A}_{2 S}$ (heavy solid), $\mathcal{A}=6.60$ (solid), $\mathcal{A}=6.90$ (dotted), and $\mathcal{A}=7.20$ (widely spaced dots). Top right: $S B B: \mathcal{A}=\mathcal{A}_{3 S}$ (heavy solid), $\mathcal{A}=9.90$ (solid), $\mathcal{A}=10.40$ (dotted), and $\mathcal{A}=11.10$ (widely spaced dots). Middle left: $S S B: \mathcal{A}=\mathcal{A}_{3 S}$ (heavy solid), $\mathcal{A}=9.020$ (solid), $\mathcal{A}=8.90$ (dotted), and $\mathcal{A}=9.04$ (widely spaced dots). Middle right: $S B B B: \mathcal{A}=\mathcal{A}_{4 S}$ (heavy solid), $\mathcal{A}=15.30$ (solid), $\mathcal{A}=16.43$ (dotted), and $\mathcal{A}=17.83$ (widely spaced dots). Bottom left: $S S B B: \mathcal{A}=\mathcal{A}_{4 S}$ (heavy solid), $\mathcal{A}=13.60$ (solid), $\mathcal{A}=13.95$ (dotted), and $\mathcal{A}=14.37$ (widely spaced dots). Bottom right: $S S S B: \mathcal{A}=\mathcal{A}_{4 S}$ (heavy solid), $\mathcal{A}=12.34$ (solid), $\mathcal{A}=11.30$ (dotted), and $\mathcal{A}=11.12$ (widely spaced dots).

\section{A traveling-wave, or drift, instability for $\tau=O\left(\varepsilon^{-2}\right)$}

In $\$ 3$ we analyzed the small eigenvalues for $\tau=O(1)$. In this section, we study the initiation of a traveling-wave, or drift, instability for a one-spike large equilibrium solution $u_{-}, v_{-}$centered at 
the origin for the regime where $\tau \gg 1$. For this solution, we show that a small eigenvalue can become complex when $\tau \gg 1$. In addition, we derive a formula for the critical value $\tau_{T W}$ of $\tau$, with $\tau_{T W} \gg 1$, where a traveling-wave instability occurs as a result of a pair of complex small eigenvalues entering the unstable right half-plane through a Hopf bifurcation. The path of these small eigenvalues as a function of $\tau$ for $\tau>\tau_{T W}$ is analyzed. This instability with respect to translations in the spike profile leads to oscillations in the spike location, and is distinct from the Hopf bifurcation of $\$ 2$ for the amplitude of the spike profile. The thresholds for drift and profile instabilities are compared, and it is shown that a drift instability is the dominant instability on the asymptotic subrange $O\left(\varepsilon^{-1 / 3}\right) \ll \mathcal{A} \ll O\left(\varepsilon^{-1 / 2}\right)$ of the intermediate regime, and is subdominant to the profile instability when $O(1) \ll \mathcal{A} \ll O\left(\varepsilon^{-1 / 3}\right)$. In the context of the infinite-line problem this interchange in the dominant instability mechanism was explored in [28].

For $\tau=O\left(\varepsilon^{-2}\right)$, we now derive a formula for the small eigenvalue associated with a one-spike solution. By repeating the analysis leading to Principal Result 3.1, we obtain the following result:

PRINCIPAL RESUlT 5.1 Let $\varepsilon \ll 1$ and $\tau=O\left(\varepsilon^{-2}\right)$. Then the small eigenvalue of 2.13 for a one-spike large solution satisfies

$$
\lambda \sim 2 \varepsilon^{2} s\left[\left\langle\tilde{\eta}_{0 x}\right\rangle_{0}-\frac{1}{D}\right] .
$$

Here $\left\langle\tilde{\eta}_{0 x}\right\rangle_{0}$ is to be calculated from the auxiliary problem

$$
\begin{gathered}
D \tilde{\eta}_{0 x x}-[1+\tau \lambda] \tilde{\eta}_{0}=0, \quad-1<x<1 ; \quad \tilde{\eta}_{0 x}( \pm 1)=0, \\
{\left[D \tilde{\eta}_{0}\right]_{0}=-\frac{1}{a_{g}} ; \quad\left[D \tilde{\eta}_{0 x}\right]_{0}=\frac{-s}{a_{g}}\left\langle\tilde{\eta}_{0}\right\rangle_{0} .}
\end{gathered}
$$

Here $\langle\zeta\rangle_{0} \equiv \frac{1}{2}\left(\zeta\left(0^{+}\right)+\zeta\left(0^{-}\right)\right),[\zeta]_{0} \equiv \zeta\left(0^{+}\right)-\zeta\left(0^{-}\right), s=\left(1-U_{-}\right) / U_{-}, a_{g}=\left[2 \sqrt{D} \tanh \theta_{0}\right]^{-1}$, and $U_{-}$is given in 2.9 .

An eigenvalue with $\operatorname{Re}(\lambda)>0$ yields a drift instability since, from 3.5 , the perturbation in $v$ is

$$
\nu_{-}(x) \sim \frac{1}{\mathcal{A} U_{-}}\left[w\left(\varepsilon^{-1} x\right)+\delta e^{\lambda t} w^{\prime}\left(\varepsilon^{-1} x\right)\right] \sim \frac{1}{\mathcal{A} U_{-}} w\left(\varepsilon^{-1}\left[x-x_{0}(t)\right]\right), \quad x_{0} \sim-\varepsilon \delta e^{\lambda t},
$$

where $\delta \ll 1$. An instability with $\lambda>0$ real leads to a monotonic drift of the spike away from $x=0$. An instability with $\lambda= \pm i \lambda_{I}$ and $\lambda_{I}>0$ leads to the onset of small-scale oscillations around $x=0$.

To determine an explicit formula for $\lambda$ in 5.1 , we must calculate $\left\langle\tilde{\eta}_{0 x}\right\rangle_{0}$. By solving 5.2 we obtain

$$
\left\langle\tilde{\eta}_{0 x}\right\rangle_{0}=\frac{\beta}{D} \tanh \left(\theta_{0} \beta\right) \tanh \theta_{0}, \quad \beta \equiv \sqrt{1+\tau \lambda} .
$$

Substituting 5.4 into (5.1), we get

$$
\lambda \sim \frac{2 \varepsilon^{2} s}{D}\left[\beta \tanh \left(\theta_{0} \beta\right) \tanh \theta_{0}-1\right] .
$$

To analyze 5.5 , it is convenient to introduce the new variables $\tau_{d}, \omega$, and $\xi$, defined by

$$
\tau=\left(\frac{D}{2 s \varepsilon^{2}}\right) \tau_{d}, \quad \lambda=\left(\frac{2 s \varepsilon^{2}}{D}\right) \omega, \quad \xi=\tau_{d} \omega=\tau \lambda
$$


Substituting 5.6 into 5.5 , we find that $\xi$ satisfies $F(\xi)=0$, where

$$
F(\xi) \equiv \frac{\xi}{\tau_{d}}-G(\xi), \quad G(\xi) \equiv \beta \tanh \theta_{0} \tanh \left(\theta_{0} \beta\right)-1, \quad \beta \equiv \sqrt{1+\xi}, \quad \theta_{0}=D^{-1 / 2} .
$$

In (5.7), the principal value of the square root is taken. In terms of the roots $F(\xi)=0$, the scaled eigenvalues $\omega$ are recovered from $\omega=\xi / \tau_{d}$.

We seek complex roots $\xi=\xi_{R}+i \xi_{I}$ to $F(\xi)=0$. For each $D>0$, we will show that $F(\xi)=0$ has a pair of complex conjugate roots on the imaginary axis $\xi_{R}=0$ when $\tau_{d}=\tau_{d h}$. Increasing $\tau_{d}$ past $\tau_{d h}$, this complex conjugate pair of roots merges onto the positive real axis in the $\xi$ plane when $\tau_{d}=\tau_{d m}$. At this value of $\tau_{d}, 5.7$ has a double real root. For $\tau_{d}>\tau_{d m}$, 5.7) always has two positive real roots.

Before analyzing the zeros of $F(\xi)$ in 5.7), we discuss the implications for the stability of a one-spike solution in the intermediate regime $O(1) \ll \mathcal{A} \ll O\left(\varepsilon^{-1 / 2}\right)$ as $\tau$ is increased. Since $s=O\left(\mathcal{A}^{2}\right)$ in this regime, 5.6 shows that a traveling-wave instability occurs when $\tau=\tau_{T W}=$ $O\left(\mathcal{A}^{-2} \varepsilon^{-2}\right)$. In contrast, from A.7) a Hopf bifurcation in the spike profile will occur when $\tau=$ $\tau_{H}=O\left(\mathcal{A}^{4}\right)$. Comparing the asymptotic orders of these two scales, we conclude that

$$
\tau_{H} \ll \tau_{T W} \quad \text { for } 1 \ll \mathcal{A} \ll O\left(\varepsilon^{-1 / 3}\right) ; \quad \tau_{T W} \ll \tau_{H} \quad \text { for } O\left(\varepsilon^{-1 / 3}\right) \ll \mathcal{A} \ll O\left(\varepsilon^{-1 / 2}\right) .
$$

For the infinite-line problem, (5.8) was also observed in [28]. Therefore, there is some scaling regime within the intermediate range $O(1) \ll \mathcal{A} \ll O\left(\varepsilon^{-1 / 2}\right)$ where, as $\tau$ is increased, a travelingwave instability will occur before the onset of the Hopf bifurcation associated with the spike profile.

We first look for roots of $F(\xi)=0$ along the real axis. These roots correspond to the intersections points of the line $\xi / \tau_{d}$ with $G(\xi)$. A simple calculation using (5.7) shows that

$$
\begin{aligned}
G_{\xi}(\xi) & =\frac{\tanh \theta_{0}}{2 \beta}\left[\tanh \left(\theta_{0} \beta\right)+\beta \theta_{0} \operatorname{sech}^{2}\left(\theta_{0} \beta\right)\right], \\
G_{\xi \xi}(\xi) & =\frac{\tanh \theta_{0}}{4 \beta^{3}}\left[\left(\theta_{0} \beta\right) \operatorname{sech}^{2}\left(\theta_{0} \beta\right)-\tanh \left(\theta_{0} \beta\right)\right]-\frac{\theta_{0}^{2}}{2 \beta} \tanh \theta_{0} \tanh \left(\theta_{0} \beta\right) \operatorname{sech}^{2}\left(\theta_{0} \beta\right) .
\end{aligned}
$$

Since $z \operatorname{sech}^{2} z-\tanh z<0$ for all $z>0$, it follows that $G(\xi)$ is an increasing, and concave, function for $\xi \geqslant 0$. In addition, $G(0)=-\operatorname{sech}^{2} \theta_{0}<0$. Therefore, there are exactly two real roots to $F(\xi)=0$ when $\tau_{d}>\tau_{d m}$ and no such roots when $0<\tau_{d}<\tau_{d m}$. Here $\tau_{d m}$ is the value of $\tau_{d}$ for which the straight line $\xi / \tau_{d}$ intersects $G(\xi)$ tangentially at some point $\xi_{m}$. By combining the equations $F=F_{\xi}=0$ for the double root at $\tau_{d}=\tau_{d m}$ and $\xi=\xi_{m}$, we find, after a little algebra, that $\xi_{m}$ is the unique root of

$$
\left(\beta^{2}+1\right) \frac{\tanh \left(\theta_{0} \beta\right)}{\theta_{0} \beta}-\left(\beta^{2}-1\right) \operatorname{sech}^{2}\left(\theta_{0} \beta\right)=\frac{2}{\theta_{0} \tanh \theta_{0}}, \quad \beta=\sqrt{1+\xi} .
$$

This yields $\xi_{m}$ as a function of $\theta_{0}=D^{-1 / 2}$, which can be computed numerically using Newton's method. In terms of $\xi_{m}$, we then calculate $\tau_{d m}$ and the eigenvalue $\omega_{m}$ from $\tau_{d m}=\xi_{m} / G\left(\xi_{m}\right)$ and $\omega_{m}=\xi_{m} / \tau_{d m}$.

In Fig. 8(a) we plot the numerical results for $\tau_{d m}$ and $\omega_{m}$ versus $D$. For $D=0.75$ and $D=0.1$, we get

$$
D=0.75, \quad \tau_{d m}=4.914, \quad \omega_{m}=0.717 ; \quad D=0.1, \quad \tau_{d m}=2.229, \quad \omega_{m}=0.116 .
$$




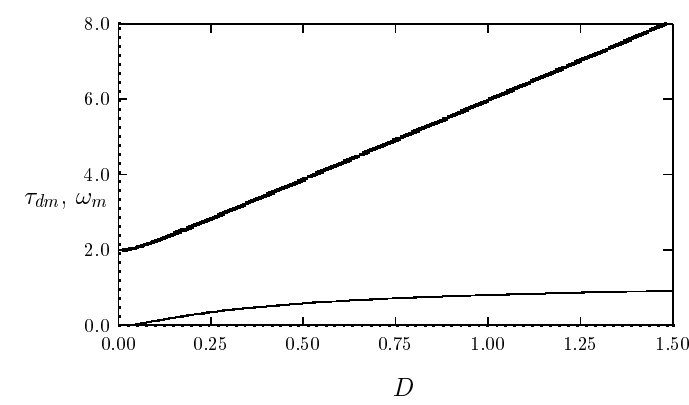

(a) $\tau_{d m}$ and $\omega_{m}$

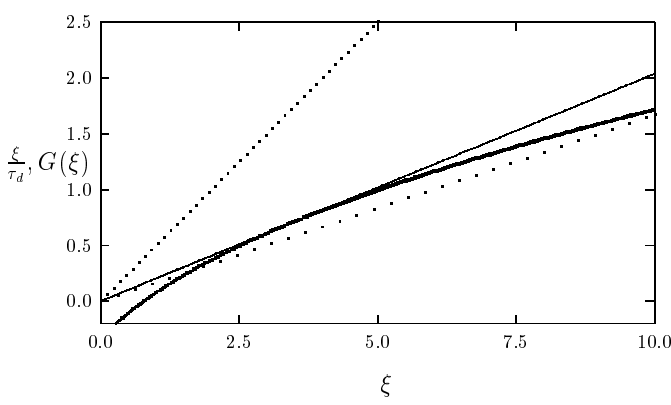

(b) $G$ versus $\xi$

FIG. 8. Left figure: $\tau_{d m}$ (heavy solid curve) and $\omega_{m}$ (solid curve) versus $D$. Right figure: graphical determination of real roots to $F=0$ when $D=0.75$; here $G(\xi)$ (heavy solid curve), and $\xi / \tau_{d}$ are shown for $\tau_{d}=2.0$ (dotted curve), $\tau_{d}=\tau_{d m} \approx 4.91$ (solid curve), and $\tau_{d}=6.0$ (widely spaced dots).

For $D=0.75$, in Fig. $8(\mathrm{~b})$ we plot $G(\xi)$ together with the straight line $\xi / \tau_{d}$ for three different values of $\tau_{d}$, one of which is the double root value $\tau_{d}=\tau_{d m}$. From this figure we see that there are two real roots to $F(\xi)=0$ when $\tau_{d}>\tau_{d m}$ and none when $0<\tau_{d}<\tau_{d m}$. Fig. 8(a) suggests that $\tau_{d m}$ and $\omega_{m}$ have limiting values as $D \rightarrow 0$ and $D \rightarrow \infty$, respectively. Let $D \rightarrow 0$ so that $\theta_{0} \rightarrow \infty$. In this limit, $\xi_{m} \rightarrow 0$ from 5.10 . Therefore, for $D \rightarrow 0, \tau_{d m}$ corresponds to the value of $\tau_{d}$ for which $F(\xi)=0$ has a double root at the origin. Using $(5.7)$, we set $F(0)=F_{\xi}(0)=0$, and let $\theta_{0} \rightarrow \infty$ to obtain

$$
\frac{1}{\tau_{d m}}=\frac{1}{2}\left[\tanh ^{2} \theta_{0}+\theta_{0} \tanh \theta_{0} \operatorname{sech}^{2} \theta_{0}\right] \rightarrow \frac{1}{2} \quad \text { as } \theta_{0} \rightarrow \infty .
$$

Hence, $\tau_{d m} \rightarrow 2$ as $D \rightarrow 0$, which is consistent with Fig. 8(a). To determine the limiting behavior of $\omega_{m}$ as $D \rightarrow \infty$, we let $\theta_{0} \rightarrow 0$ in 5.10 to conclude that $\xi_{m} \rightarrow \infty$. Since $\beta \sim \sqrt{\xi}$ when $\xi \gg 1$, the double root condition for $F$ can be written asymptotically as

$$
\frac{\xi}{\tau_{d}} \sim \sqrt{\xi} \theta_{0} \tanh \left(\sqrt{\xi} \theta_{0}\right)-1, \quad \frac{1}{\tau_{d}} \sim \frac{1}{2 \sqrt{\xi}}\left[\theta_{0} \tanh \left(\sqrt{\xi} \theta_{0}\right)+\sqrt{\xi} \theta_{0}^{2} \operatorname{sech}^{2}\left(\sqrt{\xi} \theta_{0}\right)\right] .
$$

Assuming that $\sqrt{\xi} \theta_{0} \rightarrow \mu$ as $\theta_{0} \rightarrow 0$ for some $\mu=O(1)$, we can combine the equations in 5.13, to get

$$
\mu \tanh \mu-2=\mu^{2} \operatorname{sech}^{2} \mu .
$$

The unique root of 5.14 is $\mu=2.2649$. Then, since $\omega_{m}=\xi_{m} / \tau_{d m}$ and $\xi_{m} \sim D \mu^{2}$, we conclude that

$$
\omega_{m} \sim \mu \tanh \mu-1 \approx 1.2166, \quad \xi_{m} \sim 5.1298 D, \quad \tau_{d m} \sim 4.2165 D \quad \text { as } D \rightarrow \infty .
$$

Hence for $D \gg 1, \tau_{d m}$ is linear in $D$. The limiting behavior for $\omega_{m}$ is clearly seen in Fig. 8(a)

Next, we look for roots of $F(\xi)=0$ on the positive imaginary axis $\xi=i \xi_{I}$ with $\xi_{I} \geqslant 0$. Separating (5.7) into real and imaginary parts, we obtain

$$
F_{R}\left(\xi_{I}\right)=-G_{R}\left(\xi_{I}\right), \quad F_{I}\left(\xi_{I}\right)=\frac{\xi_{I}}{\tau_{d}}-G_{I}\left(\xi_{I}\right)
$$


where $F_{R}\left(\xi_{I}\right) \equiv \operatorname{Re}\left[F\left(i \xi_{I}\right)\right], F_{I}\left(\xi_{I}\right) \equiv \operatorname{Im}\left[F\left(i \xi_{I}\right)\right], G_{R}\left(\xi_{I}\right) \equiv \operatorname{Re}\left[G\left(i \xi_{I}\right)\right]$, and $G_{I}\left(\xi_{I}\right) \equiv$ $\operatorname{Re}\left[G\left(i \xi_{I}\right)\right]$.

Using (5.7), we readily see that for each $D>0$ we have $G_{R}(0)=-\operatorname{sech}^{2} \theta_{0}<0$ and that $G_{R}\left(\xi_{I}\right)$ is increasing with $G_{R} \rightarrow+\infty$ as $\xi_{I} \rightarrow \infty$. Therefore, $F_{R}\left(\xi_{I}\right)=0$ has a unique root, which we label by $\xi_{h}$. Then, setting $F_{I}\left(\xi_{h}\right)=0$, we determine the Hopf bifurcation value of $\tau_{d}$ as

$$
\tau_{d h}=\left(\frac{\theta_{0}}{\tanh \theta_{0}}\right) \frac{\xi_{h}}{\operatorname{Im}(z \tanh z)}, \quad z \equiv \theta_{0} \sqrt{1+i \xi_{h}} .
$$

The corresponding value of $\omega$ for the frequency of small-scale oscillations is $\omega_{h}=\xi_{h} / \tau_{d h}$. In Fig. 9(a) we plot the numerical results for $\tau_{d h}$ and $\omega_{m}$ versus $D$. For $D=0.75$ and $D=0.1$, we get

$$
D=0.75, \quad \tau_{d h}=2.617, \quad \omega_{h}=0.772 ; \quad D=0.1, \quad \tau_{d h}=1.986, \quad \omega_{h}=0.115 .
$$

Clearly, $\tau_{d h} \sim \tau_{d m} \sim 2$ as $D \rightarrow 0$.

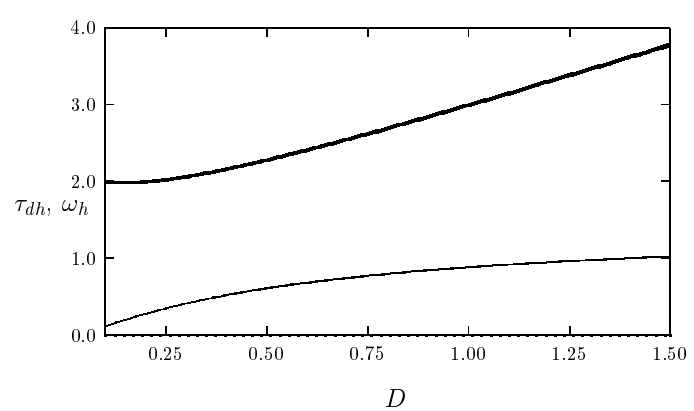

(a) $\tau_{d h}$ and $\omega_{h}$



(b) Eigenvalues in right half-plane

FIG. 9. Left figure: The Hopf bifurcation values: $\tau_{d h}$ (heavy solid curve) and $\omega_{h}$ (solid curve) versus $D$. Right figure: eigenvalues $\omega=\omega_{R}+i \omega_{I}$ in the right half-plane for $\tau>\tau_{d h}$ when $D=0.75$ (heavy solid curve) and $D=0.1$ (solid curve).

By calculating the roots of $F(\xi)=0$ with $\xi=\xi_{R}+i \xi_{I}$ numerically on the range $\tau_{d h}<\tau_{d}<$ $\tau_{d m}$ we obtain a path in the complex $\xi$ plane. In Fig. 9(b) we plot this path in terms of $\omega=\xi / \tau_{d}$ for both $D=0.75$ and $D=0.1$. These numerical results suggest that for each $\tau_{d}>\tau_{d h}$ there are exactly two small eigenvalues in the right half-plane.

To prove that, for each $D>0$, there are exactly two small eigenvalues in the right half-plane when $\tau_{d}>\tau_{d h}$ we must show that $F(\xi)=0$ has exactly two roots in $\operatorname{Re}(\xi)>0$ when $\tau_{d}>$ $\tau_{d h}$. This is done by calculating the winding number of $F(\xi)$ over the counterclockwise contour composed of the imaginary axis $-i R \leqslant \operatorname{Im}(\xi) \leqslant i R$ and the semi-circle $\Gamma_{R}$, given by $|\xi|=R>0$, for $\operatorname{Re}(\xi)>0$. For any $\tau_{d}>0$, 5.7) shows that $F(\xi) \sim\left(\xi / \tau_{d}\right)\left[1+O\left(\xi^{-1 / 2}\right)\right]$ as $|\xi| \rightarrow \infty$ in $\operatorname{Re}(\xi)>0$. Therefore, the change in the argument of $F(\xi)$ over $\Gamma_{R}$ as $R \rightarrow \infty$ is $\pi$. Since $F(\xi)$ is analytic in $\operatorname{Re}(\xi)>0$, we then use the argument principle, together with $F(\bar{\xi})=\overline{F(\xi)}$, to show that the number $M$ of zeros of $F(\xi)$ in $\operatorname{Re}(\xi)>0$ is

$$
M=\frac{1}{2}+\frac{1}{\pi}[\arg F]_{\Gamma_{I}}
$$


Here $[\arg F]_{\Gamma_{I}}$ denotes the change in the argument of $F(\xi)$ along the semi-infinite imaginary axis $\Gamma_{I}=i \xi_{I}, 0 \leqslant \xi_{I}<\infty$, traversed in the downwards direction.

To calculate $M$ we note that $F_{I}=O\left(\xi_{I}\right)$ and $F_{R}=O\left(\sqrt{\xi_{I}}\right)$ as $\xi_{I} \rightarrow \infty$, and that $F_{I}(0)=0$ with $F_{R}(0)>0$. Therefore, $\arg F=\pi / 2$ as $\xi_{I} \rightarrow+\infty$, and $\arg F=0$ at $\xi_{I}=0$. Since the root to $F_{R}\left(\xi_{I}\right)=0$ is unique, we conclude that $[\arg F]_{\Gamma_{I}}$ is either $3 \pi / 2$ or $-\pi / 2$ whenever $F_{I}\left(\xi_{I}\right)<0$ or $F_{I}\left(\xi_{I}\right)>0$, respectively, at the unique root of $F_{R}\left(\xi_{I}\right)=0$. Therefore, $M=0$ or $M=2$ from 5.19). Finally, since the root $\xi_{h}$ to $F_{R}\left(\xi_{I}\right)=0$ is independent of $\tau_{d}$, we conclude that $F_{I}\left(\xi_{I}\right)<0$ when $\tau_{d}>\tau_{d h}$ and $F_{I}\left(\xi_{I}\right)>0$ when $\tau_{d}<\tau_{d h}$. This leads to a strict transversal crossing condition at the Hopf bifurcation point. This result is illustrated in Fig.10(a) and Fig. 10(b) where we show a graphical determination of the roots of $F_{R}\left(\xi_{I}\right)=0$ and $F_{I}\left(\xi_{I}\right)=0$.

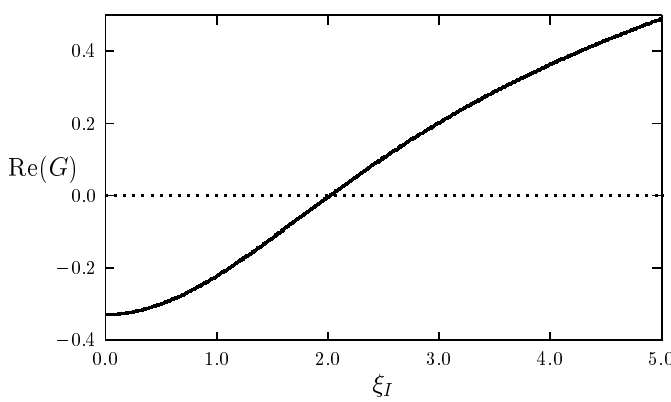

(a) $\operatorname{Re}\left(G\left(i \xi_{I}\right)\right)$ versus $\xi_{I}$

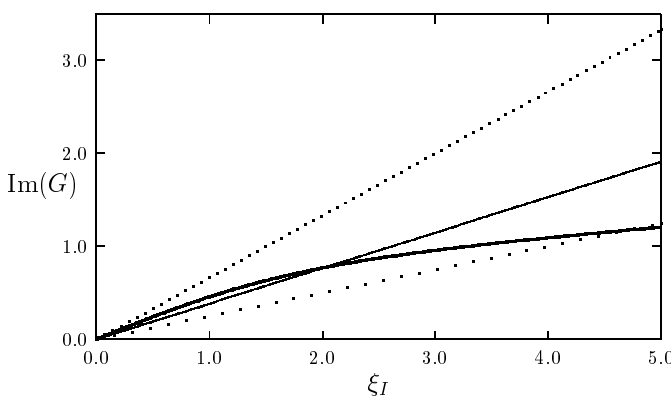

(b) $\operatorname{Im}\left(G\left(i \xi_{I}\right)\right)$ versus $\xi_{I}$

FIG. 10. Graphical determination of the roots of $F\left(i \xi_{I}\right)=0$ when $D=0.75$. Left figure: $\operatorname{Re}\left[G\left(i \xi_{I}\right)\right]$ versus $\xi_{I}$. Right figure: $\operatorname{Im}\left[G\left(i \xi_{I}\right)\right]$ versus $\xi_{I}$ (heavy solid curve). We also plot $\xi_{I} / \tau_{d}$ for $\tau_{d}=1.5$ (dotted curve), $\tau_{d}=\tau_{d h}=2.6169$ (solid curve), and $\tau_{d}=4.0$ (widely spaced dots).

We summarize our results as follows:

Proposition 5.2 Let $\varepsilon \ll 1$ and $\tau=O\left(\varepsilon^{-2}\right)$, and consider the small eigenvalue with $\lambda=$ $O\left(\varepsilon^{2}\right)$ for the large one-spike solution. Then there is a complex conjugate pair of pure imaginary eigenvalues when $\tau_{d}=\tau_{d h}$. For any $\tau_{d}>\tau_{d h}$ there are exactly two eigenvalues in the right halfplane. These eigenvalues have nonzero imaginary parts when $\tau_{d h}<\tau_{d}<\tau_{d m}$, and they merge onto the positive real axis at $\tau_{d}=\tau_{d m}$. They remain on the positive real axis for all $\tau_{d}>\tau_{d m}$.

We now compare the two thresholds of instability for a one-spike solution in the intermediate regime. Let $\tau_{H}$ denote the Hopf bifurcation threshold of the spike amplitude, and let $\tau_{T W}$ denote the drift stability threshold for a spike-layer oscillation. Then, from (A.7) and (5.6), we obtain

$$
\tau_{H}=1.748 \tanh ^{2}\left(\theta_{0}\right) s^{2}, \quad \tau_{T W}=\frac{D}{2 s \varepsilon^{2}} \tau_{d h},
$$

where

$$
s=\frac{1-U_{-}}{U_{-}}, \quad U_{-}=\frac{1}{2}\left[1-\sqrt{1-\frac{\mathcal{A}_{1 e}^{2}}{\mathcal{A}^{2}}}\right], \quad \mathcal{A}=\varepsilon^{-1 / 2} A, \quad \mathcal{A}_{1 e} \equiv \sqrt{\frac{12 \theta_{0}}{\tanh \left(\theta_{0}\right)}},
$$

and $\theta_{0} \equiv D^{-1 / 2}$. For $D=0.1$ and $D=0.75$, we plot $\log _{10}\left(\tau_{H}\right)$ and $\log _{10}\left(\tau_{T W}\right)$ in Fig. 11(a) and Fig. $11(\mathrm{~b})$ respectively, for two values of $\varepsilon$ on the parameter range $\varepsilon^{1 / 2} \mathcal{A}_{1 e}<A<1$. We 


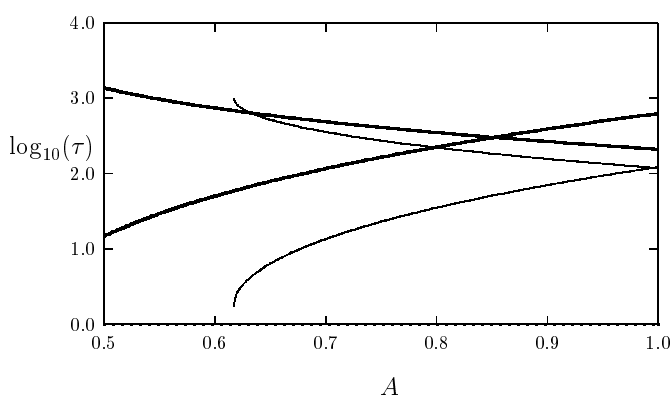

(a) $\log _{10}(\tau)$ for $D=0.1$

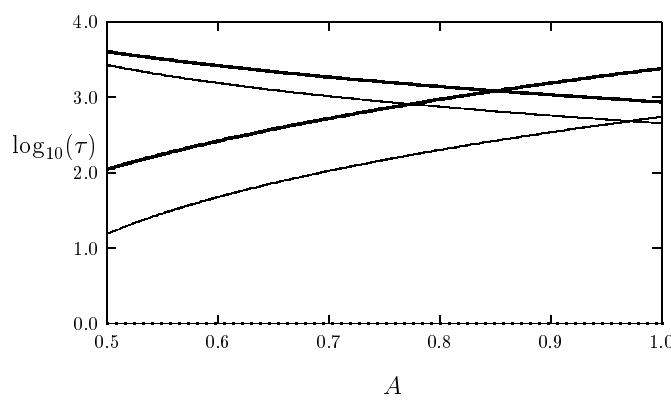

(b) $\log _{10}(\tau)$ for $D=0.75$

FIG. 11. Plots of $\log _{10}\left(\tau_{H}\right)$ (increasing curves) and $\log _{10}\left(\tau_{T W}\right)$ (decreasing curves) as a function of $A$ on the range $\varepsilon^{1 / 2} \mathcal{A}_{1 e}<A<1$. The left figure and right figure are for $D=0.1$ and $D=0.75$, respectively. The heavy solid curves are for $\varepsilon=0.005$ and the solid curves are for $\varepsilon=0.01$.

observe that when $\varepsilon$ is sufficiently small, the curves $\tau_{H}$ and $\tau_{T W}$ will cross at some point in the intermediate parameter regime. To determine this crossing point, we set $\tau_{H}=\tau_{T W}$ and solve for $A$. Using 5.20 and $s \sim 4 \varepsilon^{-1} A^{2} / \mathcal{A}_{1 e}^{2}$, we find that the de-stabilization of a one-spike solution occurs by a traveling-wave instability when

$$
A>A_{s w} \sim\left(\frac{\varepsilon D \tau_{d h}}{223.744 \tanh ^{2}\left(\theta_{0}\right)}\right)^{1 / 6} \mathcal{A}_{1 e}
$$

Alternatively, a de-stabilization by a Hopf bifurcation in the spike amplitude occurs when $A<A_{s w}$. This interchange in the instability mechanism from profile to drift instabilities as $\tau$ is increased suggests that drift instabilities will be the dominant instabilities with $\tau_{T W}=O\left(\varepsilon^{-1}\right)$ in the pulsesplitting regime where $A=O(1)$. These instabilities are indeed found to play an important role in this regime (see [22]).

We now recover the result of [29] (see Section 2.6 of [29]) for the infinite-line problem by letting $D \rightarrow 0$. In 5.21 we let $\theta_{0} \rightarrow \infty, \tau_{d h} \rightarrow 2$, and we set $\tilde{\varepsilon} \equiv \varepsilon / \sqrt{D}$ and $\mathcal{A}_{1 e}=\sqrt{12 \theta_{0}}$. Then 5.21 becomes

$$
A_{s w}=1.578 \tilde{\varepsilon}^{1 / 6}, \quad \tilde{\varepsilon} \equiv \varepsilon / \sqrt{D},
$$

which agrees with the result derived in [29]. The result (5.21) for the finite domain problem is new. Qualitatively, the effect of the finite domain on the traveling-wave instability is to de-stabilize a one-spike solution through a Hopf bifurcation leading to oscillations in the spike-layer location. This will occur for any fixed $D>0$. Alternatively, for the infinite-domain problem corresponding to the limit $D \rightarrow 0$, the unstable complex conjugate eigenvalues merge into the origin (see Fig.9(b) for a plot where $D=0.1$ ). This leads to a monotonic drift instability of a one-spike solution for the infinite-line problem.

To illustrate the analysis, we take $D=0.75, A=0.96436$, and $\varepsilon=.005$. For $D=0.75$, we get $\tau_{d h}=2.617$ from (5.18). Then, from (5.20), we calculate $\tau_{T W} \approx 935$ and $\tau_{H} \approx 2066>\tau_{T W}$. For four values of $\tau$, we then compute the numerical solution to (1.1) and output the spike location $x_{0}$ as a function of $t$. The initial condition for $v$ in (1.1) was

$$
v(x, 0)=60 \operatorname{sech}^{2}\left[\varepsilon^{-1}(x+.01)\right],
$$




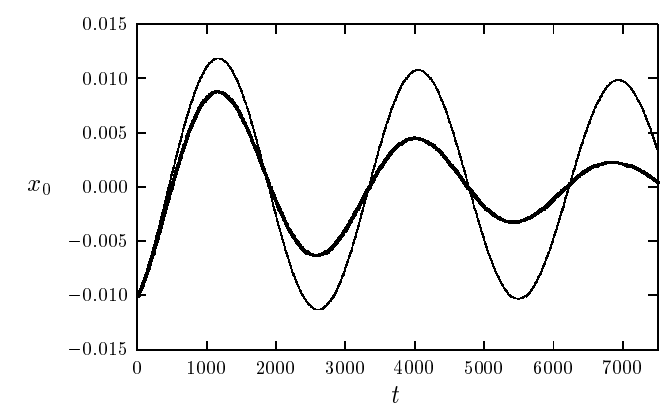

(a) $x_{0}(t)$ versus $t$

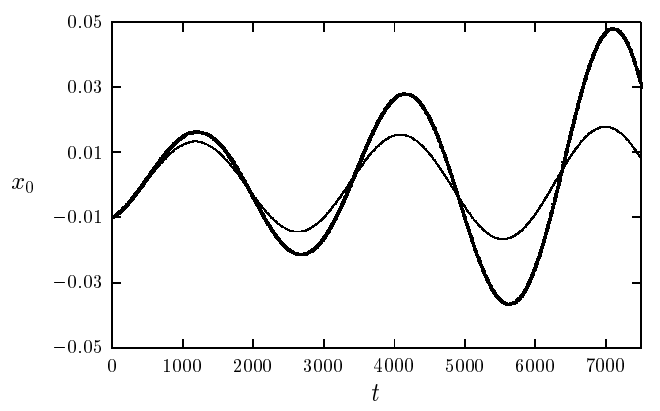

(b) $x_{0}(t)$ versus $t$

FIG. 12. Example 1: $D=0.75, A=0.96436$, and $\varepsilon=0.005$. Left figure: $x_{0}(t)$ versus $t$ for $\tau=850$ (heavy solid curve) and $\tau=920$ (solid curve). Right figure: $x_{0}(t)$ versus $t$ for $\tau=950$ (solid curve) and $\tau=1000$ (heavy solid curve). In these figures $x_{0}(t)$ is the location of the maximum of $v(x, t)$.

which represents a layer initially located at $x_{0}(0)=-0.01$. In Fig. 12(a) we show that the spike location has a decaying oscillation around $x_{0}=0$ for $\tau=850$ and $\tau=920<\tau_{T} W$. Alternatively, in Fig. 12(b), where $\tau=950>\tau_{T W}$ and $\tau=1000$, the oscillations are found to grow. The ultimate fate of these large-scale oscillations in the spike-layer location is unknown.

\section{Discussion and conclusion}

We have analyzed slow translational instabilities for two parameter regimes of the GS model (1.3). In the low feed-rate regime, we have shown analytically that $k-1$ small eigenvalues, governing the translational stability of a symmetric $k$-spike pattern, simultaneously cross through zero at precisely the same parameter value at which $k-1$ different asymmetric equilibria of the form $B S \ldots S B$ (neglecting the positioning of the $B$ and $S$ spikes in the spike sequence) bifurcate from the symmetric $k$-spike solution branch. For a one-spike equilibrium solution for $(1.3)$ in the intermediate regime $O(1) \ll \mathcal{A} \ll O\left(\varepsilon^{-1 / 2}\right)$, we showed that a traveling-wave instability will result from a Hopf bifurcation in the spike-layer location when $\tau=\tau_{T W}$, for some threshold $\tau_{T W} \gg 1$. Locally, this instability yields small-scale oscillations in the spike-layer location.

There are some specific open problems that should be investigated. The first problem is to determine the stability of asymmetric $k$-spike equilibria with respect to the large and small eigenvalues. We expect that these equilibria are all unstable with respect to translational instabilities, but that they should be stable with respect to the large $O(1)$ eigenvalues sufficiently close to the bifurcation points where they originate from the symmetric solution branches. Another open problem is to study the large-scale oscillations of the spike-layer location seen in $\S 5$ after the onset of the Hopf bifurcation that occurs when $\tau=\tau_{T W}$. The large-scale oscillations presumably arise from a local spike motion coupled to memory effects of a time-dependent Green's function. Finally, it would be interesting to study the transition between spectral properties in the weak interaction regime $D=O\left(\varepsilon^{2}\right)$ and those analyzed here in the semi-strong regime $D=O(1)$. In particular, for periodic waves of a sufficiently long wavelength it was proved in [12] that there is a continuous loop of spectra in the neighborhood of each isolated eigenvalue of the infinite-period homoclinic connection. For the GS model (1.3), this result pertains to a $k$-spike solution in the weak interaction regime $D=O\left(\varepsilon^{2}\right)$, where the spikes are separated by $O(1)$ distances. In Remark 3.1 of $\S 3$, we 
have shown that our discrete critical spectra $\lambda_{j}, j=1, \ldots, k$, for the Neumann problem, are all exponentially small when $D \ll 1$. However, our analysis is not valid when $D=O\left(\varepsilon^{2}\right)$. Therefore, it would be interesting to see how the discrete critical spectra emerge from a loop spectrum in terms of a homotopy parameter, such as $D=\varepsilon^{2-p}$ with $0 \leqslant p \leqslant 2$, which connects the weak and semi-strong interaction regimes.

In a more general context, as discussed in $\S 1$ and $\S 2$, our method of analysis is related to the SLEP method, originally developed and analyzed for transition-layer stability problems associated with two-component reaction-diffusion systems in the semi-strong limit (cf. [32]-[33]). In these systems, where the nonlinearity in the fast variable is of bistable type, the study of the critical spectrum is reduced to the study of the spectrum of a matrix eigenvalue problem (cf. [33]). Under reasonable hypotheses, the critical spectra for these problems are all negative and there are no bifurcations to other patterns (cf. [33]). In this sense, it would be worthwhile to extend the casestudy analyses presented here, and for the GM model (1.4) in [17], [45], and [47], in order to develop a "SLEP method for spikes" for general two-component systems in the semi-strong regime, where the fast component has a homoclinic connection. For this class of systems, in what sense is it generic that the critical spectra will have simultaneous zero crossings leading to asymmetric spike patterns? In this more general context, once we have derived an equivalent auxiliary problem as in Principal Result 3.1, the matrix manipulations of Appendix B could then still be used to calculate the critical spectrum explicitly.

\section{Appendix A. The instabilities of the large eigenvalues}

In this appendix we summarize some stability results for the large eigenvalues of $(2.13)$. For the low feed-rate regime, a formal asymptotic analysis was used in $\$ 3.1$ of [21] to derive a nonlocal eigenvalue problem (NLEP), which determines the stability of a $k$-spike equilibrium solution on an $O$ (1) time-scale. The result in Principal Result 3.2 of [21] is as follows:

Principal Result A.1 (from [21]) Let $\varepsilon \ll 1$ and $\mathcal{A}=O(1)$. Then, with $\Phi=\Phi(y)$, the $O(1)$ eigenvalues of (2.13) satisfy the NLEP

$$
L_{0} \Phi-\chi w^{2}\left(\frac{\int_{-\infty}^{\infty} w \Phi \mathrm{d} y}{\int_{-\infty}^{\infty} w \mathrm{~d} y}\right)=\lambda \Phi, \quad-\infty<y<\infty ; \quad \Phi \rightarrow 0 \quad \text { as }|y| \rightarrow \infty .
$$

Here $L_{0}$ is the local operator $L_{0} \Phi \equiv \Phi^{\prime \prime}-\Phi+2 w \Phi$ associated with Fisher's equation. The $k$ choices for the multiplier $\chi=\chi_{j}(z)$, and its reciprocal $C_{j}(z)$, for $j=1, \ldots, k$, are given by

$$
\left[C_{j}(\lambda)\right]^{-1} \equiv \chi_{j}(\lambda)=2 s\left(s+\frac{\sqrt{1+z}}{\tanh \left(\theta_{0} / k\right)}\left[\tanh \left(\theta_{\lambda} / k\right)+\frac{(1-\cos [\pi(j-1) / k])}{\sinh \left(2 \theta_{\lambda} / k\right)}\right]\right)^{-1},
$$

where $z \equiv \tau \lambda, \theta_{\lambda} \equiv \theta_{0} \sqrt{1+z}$, and $\theta_{0} \equiv D^{-1 / 2}$. Here $s$ is given in 2.11. There are $k$ independent choices for the vector of coefficients $\boldsymbol{b}^{t}=\left(b_{1}, \ldots, b_{k}\right)$ in 2.14, given explicitly by $\boldsymbol{b}_{j}^{t}=\left(b_{1, j}, \ldots, b_{k, j}\right)$ where

$$
\boldsymbol{b}_{1}^{t}=\frac{1}{\sqrt{k}}(1, \ldots, 1) ; \quad b_{l, j}=\sqrt{\frac{2}{k}} \cos \left(\frac{\pi(j-1)}{k}(l-1 / 2)\right), \quad j=2, \ldots, k .
$$

Here $t$ denotes transpose. 
There is an equivalent formulation of A.1. A simple calculation shows that the eigenvalues of A.1 with $\int_{-\infty}^{\infty} w \Phi \mathrm{d} y \neq 0$ are the union of the zeros of the functions $g_{j}(\lambda)=0$ for $j=1, \ldots, k$, where

$$
g_{j}(\lambda) \equiv C_{j}(\lambda)-f(\lambda), \quad f(\lambda) \equiv \frac{\int_{-\infty}^{\infty} w\left(L_{0}-\lambda\right)^{-1} w^{2} \mathrm{~d} y}{\int_{-\infty}^{\infty} w^{2} \mathrm{~d} y} .
$$

Since $L_{0} w=w^{2}$, the condition $C_{j}(0)=1$ implies that the NLEP problem A.1 has a zero eigenvalue. From a rigorous analysis of the zeros of $g_{j}(\lambda)$ in $\operatorname{Re}(\lambda) \geqslant 0$, the following main stability result for multi-spike solutions in the low feed-rate regime was obtained in Propositions 3.10 and 3.13 of [21]:

Proposition A.2 (from [21]) The small solution $u_{+}, v_{+}$is unstable on an $O(1)$ time-scale for any $0<s<1, k \geqslant 1$, and $D>0$. Next, let $k>1$, and consider the multi-spike large solution $u_{-}, v_{-}$, where $s>1$. For $D<D_{k L}$, or $\mathcal{A}>\mathcal{A}_{k L}$, this solution will be stable on an $O$ (1) time-scale when $0<\tau<\tau_{h L}$, for some $\tau_{h L}>0$. Alternatively, suppose that $D>D_{k L}$, or $\mathcal{A}_{k e}<\mathcal{A}<\mathcal{A}_{k L}$. Then this solution is unstable for any $\tau>0$. The thresholds $\mathcal{A}_{k L}$ and $D_{k L}$ are given explicitly by

$D_{k L} \equiv \frac{4}{k^{2}\left[\ln \left(r_{k}+\sqrt{r_{k}^{2}-1}\right)\right]^{2}}, \quad \mathcal{A}_{k L} \equiv \mathcal{A}_{k e} \frac{\left(\left(\gamma_{k} / 2\right)+2 \sinh ^{2}\left(\theta_{0} / k\right)\right)}{\left(\left[\left(\gamma_{k} / 2\right)+2 \sinh ^{2}\left(\theta_{0} / k\right)\right]^{2}-\left(\gamma_{k} / 2\right)^{2}\right)^{1 / 2}}$,

where $\gamma_{k} \equiv 1+\cos (\pi / k)$ and $r_{k} \equiv 1+\gamma_{k} /(s-1)$.

Suppose that $\mathcal{A}$ satisfies $\mathcal{A}>\mathcal{A}_{k L}$. Then, as $\tau$ increases beyond $\tau_{h L}$, a Hopf bifurcation in the spike amplitudes was computed numerically in [21]. The threshold $\tau_{h L}$ is given by the minimum value of the set $\tau_{j}, j=1, \ldots, k$, for which $g_{j}(\lambda)=0, j=1, \ldots, k$, has complex conjugate roots on the imaginary axis. Let $\lambda= \pm i \lambda_{h}$ be the corresponding value of $\lambda$. Then, as was shown in [21], the unstable eigenfunction most typically has the form of a synchronous oscillatory instability with

$$
v \sim v_{-}+\delta e^{i \lambda_{h} t} \phi+\text { c.c, } \quad \phi(x)=\sum_{l=1}^{k} b_{l} \Phi\left[\varepsilon^{-1}\left(x-x_{l}\right)\right], \quad b_{l}=1, \quad l=1, \ldots, k .
$$

Here c.c denotes complex conjugate and $\delta \ll 1$. This eigenfunction corresponds to $\boldsymbol{b}_{1}^{t}$ in A.2

Alternatively, suppose that $\mathcal{A}_{k e}<\mathcal{A}<\mathcal{A}_{k L}$. Then, for any $\tau>0$, the dominant initial instability was shown in [21] to have the form

$$
\begin{aligned}
& v \sim \nu_{-}+\delta e^{\lambda_{R k} t} \phi \\
& \phi(x)=\sum_{l=1}^{k} b_{l, k} \Phi\left[\varepsilon^{-1}\left(x-x_{l}\right)\right], \quad b_{l, k}=\cos \left(\frac{\pi(k-1)}{k}(l-1 / 2)\right), \quad l=1, \ldots, k .
\end{aligned}
$$

Here $\delta \ll 1$, and $\lambda_{R k}>0$ is the unique root of $g_{k}\left(\lambda_{R}\right)=0$. This form corresponds to the choice $\boldsymbol{b}_{k}$ in A.2). Since $\sum_{l=1}^{k} b_{l, k}=0$, this instability locally conserves the sum of the heights of the spikes. Hence, it is referred to as a competition instability. The numerical experiments in $\S 3.3$ of [21] show that this instability leads to a spike competition process whereby certain spikes in a spike sequence are ultimately annihilated. The first qualitative discussion of competition instabilities for other systems, referred to there as activator re-pumping, was given in [19] and [18] (see Sections 14.4.8, 15.3, Fig. 14.13, and Fig. 14.16 of [18]). 
In the intermediate regime $O(1) \ll \mathcal{A} \ll O\left(\varepsilon^{-1 / 2}\right)$, corresponding to $s \rightarrow \infty$ in $\sqrt{2.11}$, the stability results for symmetric $k$-spike patterns simplify considerably. By letting $s \rightarrow \infty$ in A.1, an instability occurs only when $\tau \gg 1$. In this way, the following result can be obtained:

Principal Result A.3 (from [21]) Let $\varepsilon \ll 1, D=O(1)$, and $O(1) \ll \mathcal{A} \ll O\left(\varepsilon^{-1 / 2}\right)$. Then the $k$-spike equilibrium large solution $u_{-}, v_{-}$is stable on an $O(1)$ time-scale when $\tau<\tau_{H}$, where $\tau_{H}=O\left(\mathcal{A}^{4}\right)$ is given by

$$
\tau_{H} \sim \frac{\mathcal{A}^{4} D}{9} \tanh ^{4}\left(\theta_{0} / k\right) \tau_{0 h}\left(1-\frac{6 \theta_{0}}{\mathcal{A}^{2} \tanh \left(\theta_{0} / k\right)}\right)^{2}+o(1),
$$

and $\tau_{0 h}=1.748$. As $\tau$ increases past $\tau_{H}$, stability is lost due to a Hopf bifurcation. The critical value for $\tau_{0 h}$ is the minimum value of $\tau_{0}$ for which the following NLEP has eigenvalues on the imaginary axis:

$$
L_{0} \Phi-\frac{2 w^{2}}{1+\sqrt{\tau_{0} \lambda}}\left(\frac{\int_{-\infty}^{\infty} w \Phi \mathrm{d} y}{\int_{-\infty}^{\infty} w \mathrm{~d} y}\right)=\lambda \Phi, \quad-\infty<y<\infty ; \quad \Phi \rightarrow 0 \quad \text { as }|y| \rightarrow \infty .
$$

The result A.7 is asymptotically equivalent to the stability result in the intermediate regime first derived in equation (5.16) of [7] in terms of an alternative dimensionless form of the GS model. In the form (A.7), this result was reported in Principal Result 4.2 and Proposition 4.3 of [21]. A rigorous analysis of (A.8) was given in [8], with an alternative analysis given in $\$ 4$ of [21]. In the intermediate regime, competition instabilities for multi-spike solutions can only occur if $D \gg 1$, or equivalently if the inter-spike separation distance $L=2 / k$ is too small. The result, as obtained in $\S 4$ of [21], is given in (3.42) above.

\section{Appendix B. Matrix calculations of the small eigenvalues}

In this appendix we give a brief outline of the derivation of the explicit formulae in Proposition 3.2 for the small eigenvalues. The solution to 3.25$]$ is decomposed as

$$
\tilde{\eta}_{0}(x)=\frac{1}{a_{g}}\left(\sum_{j=1}^{k} c_{j} g\left(x ; x_{j}\right)+\sum_{j=1}^{k} m_{j} G\left(x ; x_{j}\right)\right),
$$

for some unknown coefficients $m_{j}$, for $j=1, \ldots, k$. Here $G\left(x ; x_{j}\right)$ is the Green's function satisfying 2.5 , while $g\left(x ; x_{j}\right)$ is the dipole Green's function satisfying

$$
D g_{x x}-g=-\delta^{\prime}\left(x-x_{j}\right), \quad-1<x<1 ; \quad g_{x}\left( \pm 1 ; x_{j}\right)=0 .
$$

Define the vectors $\boldsymbol{m}^{t} \equiv\left(m_{1}, \ldots, m_{k}\right)$ and $\boldsymbol{c}^{t} \equiv\left(c_{1}, \ldots, c_{k}\right)$, where $t$ denotes transpose. Then, by satisfying the jump conditions in $3.25 \mathrm{~b}$, we get a matrix problem for $\boldsymbol{m}$ in terms of $\boldsymbol{c}$ of the form

$$
\left(-\frac{s}{a_{g}} \mathcal{G}+I\right) \boldsymbol{m}=\frac{s}{a_{g}} \mathcal{P}_{g} \mathbf{c} .
$$

Here $\mathcal{G}$ and $\mathcal{P}_{g}$ are matrices associated with $G$ and $g$, defined by

$$
\mathcal{G} \equiv\left(\begin{array}{ccc}
G\left(x_{1} ; x_{1}\right) & \cdots & G\left(x_{1} ; x_{k}\right) \\
\vdots & \ddots & \vdots \\
G\left(x_{k} ; x_{1}\right) & \cdots & G\left(x_{k} ; x_{k}\right)
\end{array}\right), \quad \mathcal{P}_{g} \equiv\left(\begin{array}{ccc}
\left\langle g\left(x_{1} ; x_{1}\right)\right\rangle_{1} & \cdots & g\left(x_{1} ; x_{k}\right) \\
\vdots & \ddots & \vdots \\
g\left(x_{k} ; x_{1}\right) & \cdots & \left\langle g\left(x_{k} ; x_{k}\right)\right\rangle_{k}
\end{array}\right)
$$

As in $\S 3$, the angle brackets in (B.4) again denote the average of the right and left-sided limits. 
In 3.24 we must calculate $\left\langle\tilde{\boldsymbol{\eta}}_{0 x}\right\rangle^{t} \equiv\left(\left\langle\tilde{\eta}_{0 x}\right\rangle_{1}, \ldots,\left\langle\tilde{\eta}_{0 x}\right\rangle_{k}\right)$. To do so, we use B.1] to get

$$
\left\langle\tilde{\boldsymbol{\eta}}_{0 x}\right\rangle=\frac{1}{a_{g}}\left(\mathcal{G}_{g} \boldsymbol{c}+\mathcal{P} \boldsymbol{m}\right),
$$

where $\mathcal{G}_{g}$ and $\mathcal{P}$ are two additional Green's function matrices defined by

$$
\mathcal{G}_{g} \equiv\left(\begin{array}{ccc}
g_{x}\left(x_{1} ; x_{1}\right) & \cdots & g_{x}\left(x_{1} ; x_{k}\right) \\
\vdots & \ddots & \vdots \\
g_{x}\left(x_{k} ; x_{1}\right) & \cdots & g_{x}\left(x_{k} ; x_{k}\right)
\end{array}\right), \quad \mathcal{P} \equiv\left(\begin{array}{ccc}
\left\langle G_{x}\left(x_{1} ; x_{1}\right)\right\rangle_{1} & \cdots & G_{x}\left(x_{1} ; x_{k}\right) \\
\vdots & \ddots & \vdots \\
G_{x}\left(x_{k} ; x_{1}\right) & \cdots & \left\langle G_{x}\left(x_{k} ; x_{k}\right)\right\rangle_{k}
\end{array}\right)
$$

Next, we define a new eigenvalue variable $\sigma$ in terms of $\lambda$ by

$$
\lambda=\frac{2 \varepsilon^{2} s}{a_{g}} \sigma .
$$

Combining (3.24), (B.5), and (B.7), we obtain a matrix eigenvalue problem for $\sigma$ and $c$ given by

$$
\mathcal{G}_{g} \boldsymbol{c}+\mathcal{P} \boldsymbol{m}=\left(\sigma+\frac{a_{g}}{D}\right) \boldsymbol{c},
$$

where $\boldsymbol{m}$ is determined in terms of $\boldsymbol{c}$ by $(\mathrm{B} .3)$.

In Appendices $\mathrm{A}$ and $\mathrm{C}$ of [17] it was shown that the inverses of $\mathcal{G}$ and $\mathcal{G}_{g}$ are tridiagonal matrices, which are given explicitly by

$$
\mathcal{G}=\frac{\mathcal{B}^{-1}}{\sqrt{D}}, \quad \mathcal{G}_{g}=\frac{\mathcal{B}_{g}^{-1}}{D^{3 / 2}} .
$$

Here $\mathcal{B}$ is a tridiagonal matrix defined by

$$
\mathcal{B} \equiv\left(\begin{array}{ccccccc}
d & f & 0 & \cdots & 0 & 0 & 0 \\
f & e & f & \cdots & 0 & 0 & 0 \\
0 & f & e & \ddots & 0 & 0 & 0 \\
\vdots & \vdots & \ddots & \ddots & \ddots & \vdots & \vdots \\
0 & 0 & 0 & \ddots & e & f & 0 \\
0 & 0 & 0 & \cdots & f & e & f \\
0 & 0 & 0 & \cdots & 0 & f & d
\end{array}\right),
$$

with matrix entries

$$
d \equiv \operatorname{coth}\left(2 \theta_{0} / k\right)+\tanh \left(\theta_{0} / k\right), \quad e \equiv 2 \operatorname{coth}\left(2 \theta_{0} / k\right), \quad f \equiv-\operatorname{csch}\left(2 \theta_{0} / k\right) .
$$

Here $\theta_{0} \equiv D^{-1 / 2}$. The matrix $\mathcal{B}_{g}$ has exactly the same tridiagonal form as in B.10a, except that the coefficients $d, e$, and $f$ in B.10b are to be replaced with $d_{g}, e_{g}$, and $f_{g}$, respectively, where

$$
d_{g} \equiv \operatorname{coth}\left(2 \theta_{0} / k\right)+\operatorname{coth}\left(\theta_{0} / k\right), \quad e_{g} \equiv 2 \operatorname{coth}\left(2 \theta_{0} / k\right), \quad f_{g} \equiv-\operatorname{csch}\left(2 \theta_{0} / k\right) .
$$


A key condition is that we can solve $\overline{B .3}$ for $\boldsymbol{m}$ in terms of $\boldsymbol{c}$. This requires that the matrix in

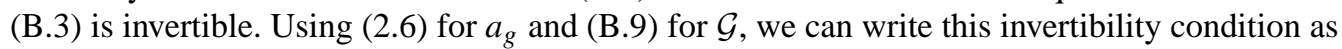

$$
s \gamma-\kappa_{j} \neq 0, \quad j=1, \ldots, k, \quad \gamma \equiv\left[a_{g} \sqrt{D}\right]^{-1}=2 \tanh \left(\theta_{0} / k\right) .
$$

Here $\kappa_{j}$ is an eigenvalue of $\mathcal{B}$. The spectra $\kappa_{j}$ and $\boldsymbol{q}_{j}$ of $\mathcal{B}$ were calculated in Proposition 2 of [17] as

$$
\begin{aligned}
& \kappa_{j}=2 \tanh \left(\theta_{0} / k\right)+2\left[1-\cos \left(\frac{\pi(j-1)}{k}\right)\right] \operatorname{csch}\left(2 \theta_{0} / k\right), \quad j=1, \ldots, k, \\
& \boldsymbol{q}_{1}^{t}=\frac{1}{\sqrt{k}}(1, \ldots, 1) ; \quad q_{l, j}=\sqrt{\frac{2}{k}} \cos \left(\frac{\pi(j-1)}{k}(l-1 / 2)\right), \quad j=2, \ldots, k .
\end{aligned}
$$

Here $\boldsymbol{q}_{j}^{t}=\left(q_{1, j}, \ldots, q_{k, j}\right)$. The invertibility condition $\overline{\mathrm{B} .12}$ has an interpretation in terms of the large eigenvalues of Appendix A. To see this, we write $C_{j}(0)$ in A.3 and A.1b in terms of $\kappa_{j}$ as

$$
C_{j}(0)=1-\frac{1}{2 s \gamma}\left(s \gamma-\kappa_{j}\right) \text {. }
$$

In Appendix A it was shown that $C_{j}(0)=1$ implies that the NLEP problem of Principal Result A.1 has a zero eigenvalue. Therefore, $[\mathrm{B} .12]$ is equivalent to the condition that the parameters $s, k$, and $D$ do not correspond to a zero large eigenvalue. Assuming that $(B .12)$ holds, we can combine $B .3$ and (B.8) to get

$$
\mathcal{G}_{g} c+\frac{s}{a_{g}} \mathcal{P}\left(-\frac{s}{a_{g}} \mathcal{G}+I\right)^{-1} \mathcal{P}_{g} c=\left(\sigma+\frac{a_{g}}{D}\right) c .
$$

To calculate $\sigma$ in B.15) explicitly, we first introduce the spectral decomposition of $\mathcal{B}$,

$$
\mathcal{B}=\mathcal{Q K} \mathcal{Q}^{t}
$$

Here $\mathcal{Q}$ is the orthogonal matrix of eigenvectors $\boldsymbol{q}_{j}$ of $\mathcal{B}$, and $\mathcal{K}$ is the diagonal matrix of eigenvalues $\kappa_{j}$. In addition, we use the following key identity of equation (4.37c) of [17]:

$$
\mathcal{P B}=-\left(\mathcal{P}_{g} \mathcal{B}_{g}\right)^{t}
$$

By substituting $(\overline{B .9}),(\overline{B .16})$, and $(\overline{B .17})$, into $(\overline{B .15})$, and assuming that the invertibility condition B.12) holds, we can derive the following generalized symmetric eigenvalue problem:

$$
\mathcal{B}_{g} \boldsymbol{u}=\omega(I+\mathcal{R}) \boldsymbol{u} .
$$

Here the symmetric matrix $\mathcal{R}$ is defined in terms of a diagonal matrix $\mathcal{D}$ by

$$
\mathcal{R} \equiv\left(\mathcal{P}_{g} \mathcal{B}_{g}\right)^{t} \mathcal{Q D} \mathcal{Q}^{t} \mathcal{P}_{g} \mathcal{B}_{g}, \quad \mathcal{D}_{j j}=\frac{s D^{2} \gamma}{s \gamma-\kappa_{j}}, \quad j=1, \ldots, k, \quad \gamma \equiv 2 \tanh \left(\frac{\theta_{0}}{k}\right) .
$$

The spectra of (B.18) and (B.15) are related by

$$
\sigma=\frac{1}{D^{3 / 2}}\left(\frac{1}{\omega}-\frac{1}{\gamma}\right), \quad c=\mathcal{B}_{g} u .
$$

Since $\mathcal{B}_{g}$ is invertible, $\omega=0$ is not an eigenvalue. 
In $\S 4.2$ of [17] (see equations (4.47)-(4.57) of [17]) it was shown that $\mathcal{R}$ and $\mathcal{B}_{g}$ are related by a similarity transformation, and so have a common set of eigenvectors. In Proposition 9 of [17] the eigenvalues $\xi_{j}$ and eigenvectors $\boldsymbol{u}_{j}$ of $\mathcal{B}_{g}$ were calculated explicitly as

$$
\begin{aligned}
& \xi_{j}=2 \operatorname{coth}\left(\frac{2 \theta_{0}}{k}\right)-2 \operatorname{csch}\left(\frac{2 \theta_{0}}{k}\right) \cos \left(\frac{\pi j}{k}\right), \quad j=1, \ldots, k, \\
& \boldsymbol{u}_{k}^{t}=\frac{1}{\sqrt{k}}\left(1,-1,1, \ldots,(-1)^{k+1}\right) ; \quad \boldsymbol{u}_{l, j}=\sqrt{\frac{2}{k}} \sin \left(\frac{\pi j}{k}(l-1 / 2)\right), \quad j=1, \ldots, k-1 .
\end{aligned}
$$

Here $\boldsymbol{u}_{j}^{t}=\left(u_{1, j}, \ldots, u_{k, j}\right)$. The matrix $\mathcal{R}$ has the same eigenvectors as $\mathcal{B}_{g}$ and its eigenvalues $z_{j}$ for $j=1, \ldots, k$ can be calculated explicitly as (see equation (4.56) of [17])

$$
z_{j}=\frac{s \gamma}{s \gamma-\xi_{j}} \operatorname{csch}^{2}\left(\frac{2 \theta_{0}}{k}\right) \sin ^{2}\left(\frac{\pi j}{k}\right), \quad j=1, \ldots, k .
$$

Therefore, $\boldsymbol{u}_{j}$ is an eigenvector of (B.18a), with eigenvalue

$$
\omega_{j}=\xi_{j} /\left(1+z_{j}\right), \quad j=1, \ldots, k,
$$

Next, we substitute (B.20a) and (B.21) into (B.22). Then, from (B.19), we can determine $\lambda_{j}$ in (B.7). The result is given in 3.26a) of Proposition 3.2.

Since $c_{j}=\mathcal{B}_{g} \boldsymbol{u}_{j}=\xi_{j} \boldsymbol{u}_{j}$, the components of each eigenvector of $\mathcal{B}_{g}$ generate a set of coefficients $c_{1}, \ldots, c_{k}$ in $3.5 \mathrm{~b}$. This is expressed in 3.27a. Finally, we calculate the $O(\varepsilon)$ term in the eigenfunction of 3.27a). From 3.10, 3.11, 3.14, and 3.23, we obtain

$$
c_{j} \phi_{1 j} \sim f\left(x_{j}\right) w_{j}=\frac{\left\langle\eta_{0}\right\rangle_{j}}{\mathcal{A} U_{ \pm}^{2}}=\frac{6 a_{g}}{\mathcal{A}^{2} U_{ \pm}^{2}}\left\langle\tilde{\eta}_{0}\right\rangle_{j}=s\left\langle\tilde{\eta}_{0}\right\rangle_{j}, \quad j=1, \ldots, k .
$$

We then use $\mathrm{B} .1$ and $\mathrm{B} .3$ to write $\left\langle\tilde{\boldsymbol{\eta}}_{0}\right\rangle^{t}=\left(\left\langle\tilde{\eta}_{0}\right\rangle_{1}, \ldots,\left\langle\tilde{\eta}_{0}\right\rangle_{k}\right)$ as

$$
\left\langle\tilde{\boldsymbol{\eta}}_{0}\right\rangle=\frac{1}{a_{g}}\left(\mathcal{P}_{g} \boldsymbol{c}+\mathcal{G} \boldsymbol{m}\right)=\frac{1}{a_{g}}\left(I+\frac{s}{a_{g}} \mathcal{G}\left(-\frac{s}{a_{g}} \mathcal{G}+I\right)^{-1}\right) \mathcal{P}_{g} \boldsymbol{c} .
$$

Then, by using $\left(\mathrm{B} .9\right.$, , we can write $\left\langle\tilde{\boldsymbol{\eta}}_{0}\right\rangle$ as in $3.27 \mathrm{~b}$.

\section{Acknowledgements}

T. K. was supported by a PGS-B graduate scholarship from NSERC (Canada). M. J. W. thanks for the grant support of NSERC, and for the hospitality of the IMS of the Chinese University of Hong Kong. J. W. thanks for the support of RGC of Hong Kong and a direct grant from CUHK.

\section{REFERENCES}

1. Alikakos, N. D., Bates, P., \& Fusco, G. Slow motion for the Cahn-Hilliard equation in one space dimension. J. Differential Equations 90 (1991), 81-135. Zbl 0753.35042 MR 1094451

2. Alikakos, N. D., Chen, X., \& Fusco, G. Motion of a droplet by surface tension along the boundary. Calc. Var. Partial Differential Equations 11 (2000), 233-305. Zbl 0994.35052 MR 1797871

3. Alikakos, N. D., Fusco, G., \& Stephanopolous, C. Critical spectrum and stability of interfaces for a class of reaction-diffusion equations. J. Differential Equations 126 (1996), 106-167. Zbl 0879.35074 MR 1382059 
4. CHEN, X. Spectrum for the Allen-Cahn, Cahn-Hilliard, and phase-field equations for generic interfaces. Comm. Partial Differential Equations 19 (1994), 1371-1395. Zbl 0811.35098 MR 1284813

5. Doelman, A., Eckhaus, W., \& Kaper, T. J. Slowly modulated two-pulse solutions in the GrayScott model I: asymptotic construction and stability. SIAM J. Appl. Math. 61 (2000), 1080-1102. Zbl 0979.35074 MR 1789392

6. Doelman, A., Eckhaus, W., \& Kaper, T. J. Slowly modulated two-pulse solutions in the GrayScott model II: geometric theory, bifurcations, and splitting dynamics. SIAM J. Appl. Math. 61 (2000), 2036-2061. Zbl 0989.35073 MR 1856881

7. Doelman, A., GARDner, R. A., \& KAPER, T. J. Stability analysis of singular patterns in the 1D GrayScott model: a matched asymptotics approach. Phys. D 122 (1998), 1-36. Zbl 0943.34039 MR 1650111

8. Doelman, A., Gardner, R. A., \& Kaper, T. J. A stability index analysis of 1-D patterns of the Gray-Scott model. Mem. Amer. Math. Soc. 155 (2002), no. 737. Zbl $0994.35059 \mid$ MR 1878337

9. Doelman, A., Gardner, R., \& Kaper, T. J. Large stable pulse solutions in reaction-diffusion systems. Indiana Univ. Math. J. 50 (2001), 443-507. Zbl 0994.35058 MR 1857043

10. Doelman, A., Kaper, T. J., \& VAn Der Ploeg, H. Spatially periodic and aperiodic multi-pulse patterns in the one-dimensional Gierer-Meinhardt equation. Methods Appl. Anal. 8 (2001), 387-414. Zbl 1006.92008 MR 1904752

11. Doelman, A., Kaper, T. J., \& Zegeling, P. Pattern formation in the one-dimensional Gray-Scott model. Nonlinearity 10 (1997), 523-563. Zbl 0905.35044 MR 1438266

12. GARDNER, R. A. Spectral analysis of long wavelength periodic waves and applications. J. Reine Angew. Math. 491 (1997), 149-181. Zbl 0883.35055 MR 1476091

13. Gierer, A., \& Meinhardt, H. A theory of biological pattern formation. Kybernetik 12 (1972), 30-39.

14. GRAY, P., \& SCOTT, S. K. Autocatalytic reactions in the isothermal, continuous stirred tank reactor: oscillations and instabilities in the system $A+2 B \rightarrow 3 B, B \rightarrow C$. Chem. Eng. Sci. 39 (1984), 1087-1097.

15. IKedA, T., \& Nishiura, Y. Pattern selection for two breathers. SIAM J. Appl. Math. 54 (1994), 195-230. Zbl 0791.35063 MR 1260914

16. IRON, D., \& WARD, M. J. The dynamics of multi-spike solutions to the one-dimensional GiererMeinhardt model. SIAM J. Appl. Math. 62 (2002), 1924-1951. Zbl 1019.35016 MR 1918301

17. Iron, D., WARD, M. J., \& WeI, J. The stability of spike solutions to the one-dimensional GiererMeinhardt model. Phys. D 150 (2001), 25-62. Zbl 0983.35020 MR 1818735

18. Kerner, B. S., \& Osipov, V. V. Autosolitons: a New Approach to Problems of Self-Organization and Turbulence. Kluwer, Dordrecht (1994). MR 1307622

19. Kerner, B. S., \& Osipov, V. V. On the spontaneous onset of irregular or pulsating structures at the stratification of the uniform state of nonequilibrium systems. Soviet Phys. Dokl. 28 (1983), 485-487.

20. Kolokolnikov, T., \& WARD, M. J. Bifurcation of spike equilibria in a near shadow reaction-diffusion system. Discrete Contin. Dyn. Syst. Ser. B 4 (2004), 1033-1064. Zbl 1063.35084 MR 2082923

21. Kolokolnikov, T., Ward, M. J., \& WeI, J. The existence and stability of spike equilibria in the one-dimensional Gray-Scott model: the low feed-rate regime. Stud. Appl. Math. 115 (2005), 21-71. MR 2141275

22. Kolokolnikov, T., Ward, M. J., \& WeI, J. The existence and stability of spike equilibria in the one-dimensional Gray-Scott model: the pulse-splitting regime. Phys. D 202 (2005), 258-293. Zbl pre02173562 MR 2132390

23. Kolokolnikov, T., WARD, M. J., \& WeI, J. Zigzag and breakup instabilities of stripes and rings in the two-dimensional Gray-Scott model. Stud. Appl. Math. 116 (2006), 35-95. MR 2201170

24. Lee, K. J., McCormick, W. D., Pearson, J. E., \& Swinney, H. L. Experimental observation of self-replicating spots in a reaction-diffusion system. Nature $\mathbf{3 6 9}$ (1994), 215-218.

25. LEe, K. J., \& SWInNey, H. L. Lamellar structures and self-replicating spots in a reaction-diffusion system. Phys. Rev. E 51 (1995), 1899-1915. 
26. Meinhardt, H. Models of Biological Pattern Formation. Academic Press, London (1982).

27. Mimura, M., \& Nishiura, N. Layer oscillations in reaction-diffusion systems. SIAM J. Appl. Math. 49 (1989), 481-514. Zbl 0691.35009 MR 0988616

28. Muratov, C., \& Osipov, V. V. Traveling spike auto-solitons in the Gray-Scott model. Phys. D 155 (2001), 112-131. Zbl 0986.34023 MR 1837206

29. Muratov, C., \& OSIPOV, V. V. Stability of the static spike autosolitons in the Gray-Scott model. SIAM J. Appl. Math. 62 (2002), 1463-1487. Zbl 1012.35042 MR 1918563

30. Muratov, C., \& Osipov, V. V. Static spike autosolitons in the Gray-Scott model. J. Phys. A: Math. Gen. 33 (2000), 8893-8916. MR 1801475

31. NAG Fortran library Mark 17, routine D03PCF, Numerical Algorithms Group Ltd., Oxford (1995).

32. NishiURA, Y., \& FUJII, H. Stability of singularly perturbed solutions to systems of reaction-diffusion equations. SIAM J. Math. Anal. 18 (1987), 1726-1770. Zbl $0638.35010 \mid$ MR 0911661

33. NishiURA, Y., \& FUJII, H. SLEP method to the stability of singularly perturbed solutions with multiple internal transition layers in reaction-diffusion systems. Dynamics of Infinite-Dimensional Systems (Lisbon, 1986), NATO Adv. Sci. Inst. Ser. F Comput. Systems Sci. 37, Springer, Berlin (1987), 211-230. Zbl 0642.35009 MR 0921913

34. Nishiura, Y., Mimura, M., IkedA, H., \& FujiI, H. Singular limit analysis of stability of traveling wave solutions in bistable reaction-diffusion systems. SIAM J. Math. Anal. 21 (1990), 85-122. Zbl 0713.35009 MR 1032729

35. Nishiura, Y., Teramoto, T., \& Ueda, K. Scattering and separators in dissipative systems. Phys. Rev. E 67 (2003), no. 5, 56210.

36. Nishiura, Y., \& Ueyama, D. A skeleton structure of self-replicating dynamics. Phys. D 130 (1999), 73-104. Zbl 0936.35090

37. Nishiura, Y., \& Ueyama, D. Spatio-temporal chaos for the Gray-Scott model. Phys. D 150 (2001), 137-162. Zbl 0981.35022

38. Pearson, J. E. Complex patterns in a simple system. Science 216 (1993), 189-192.

39. Petrov, V., Scott, S. K., \& Showalter, K. Excitability, wave reflection, and wave splitting in a cubic autocatalysis reaction-diffusion system. Philos. Trans. Roy. Soc. Lond. Ser. A 347 (1994), 631-642. Zbl 0867.35047

40. REN, X., \& WEI, J. Wriggled lamellar solutions and their stability in the diblock copolymer problem. SIAM J. Math. Anal. 37 (2005), 455-489. MR 2176111

41. Reynolds, W. N., Ponce-Dawson, S., \& Pearson, J. E. Dynamics of self-replicating spots in reaction-diffusion systems. Phys. Rev. E 56 (1997), 185-198. MR 1459091

42. Sun, W., TANG, T., WARD, M. J., \& WEI, J. Numerical challenges for resolving spike dynamics for two reaction-diffusion systems. Stud. Appl. Math. 111 (2003), 41-84. MR 1985995

43. Sun, W., WARD, M. J., \& RuSSEll, R. The slow dynamics of two-spike solutions for the Gray-Scott and Gierer-Meinhardt systems: competition and oscillatory instabilities. SIAM J. Appl. Dyn. Syst. 4 (2005), 904-953. MR 2179492

44. Ueyama, D. Dynamics of self-replicating patterns in the one-dimensional Gray-Scott model. Hokkaido Math. J. 28 (1999), 175-210. Zbl 0987.34031 MR 1673486

45. WARD, M. J., \& WEI, J. Asymmetric spike patterns for the one-dimensional Gierer-Meinhardt model: equilibria and stability. European J. Appl. Math. 13 (2002), 283-320. Zbl 1010.35002 MR 1915503

46. WARD, M. J., \& WEI, J. Hopf bifurcations and oscillatory instabilities of spike solutions for the one-dimensional Gierer-Meinhardt model. J. Nonlinear Sci. 13 (2003), 209-264. Zbl 1030.35011 MR 1978197

47. WEI, J., \& WINTER, M. Existence and stability analysis of asymmetric patterns for the Gierer-Meinhardt system. J. Math. Pures Appl. (9) 83 (2004), 433-476. Zbl pre02118456 MR 2048385 